\title{
Synthesis of vicinal dideoxy-difluorinated galactoses
}

Received 00th January 20xx, Accepted 00th January 20xx

DOI: $10.1039 / x 0 x x 00000 x$
Julien Malassis, † Jean-Baptiste Vendeville, † Qui-Hien Nguyen, Marie Boujon, Quentin Gaignard-Gaillard, Mark Light, Bruno Linclau*

Fluorinated carbohydrates have been employed as probes for fundamental studies of protein-carbohydrate interactions, but also in the development of mechanism-based enzyme inhibitors. There is a continuing demand for novel fluorinated carbohydrate probes. Whereas most examples so far involved monodeoxyfluorinated sugars, multiply deoxyfluorinated sugars have gained much interest. Here we report the synthesis and characterisation of novel vicinal dideoxy-difluorinated D-galactoses with fluorination at the 3,4-positions, and at the 2,3-positions, the latter in both the pyranose and furanose forms. This includes a successful pyranose-into-furanose isomerisation protocol.

\section{Introduction}

Fluorine incorporation in carbohydrates has been successfully employed for applications such as epitope mapping, ${ }^{1-3}$ stabilisation of glycosidic bonds, ${ }^{4}{ }^{18} \mathrm{~F}$ imaging 5,6 and mechanism-based inhibitor design. ${ }^{7-9}$ New ${ }^{19} \mathrm{~F}$ NMR based developments allow for detailed study of protein-carbohydrate interactions. ${ }^{3,10,11}$

Interes $\pi$ t in multiply deoxyfluorinated carbohydrates commenced with seminal work of Withers, ${ }^{1}$ in which he reported that 1,2-dideoxy-1,2-difluorinated glucose derivatives 1 and $\mathbf{2}$ (Figure 1) displayed a larger affinity to glycogen phosphorylase than what could be expected from the values of their respective monofluorinated derivatives. Furthermore, it was shown that the stereochemistry at the fluorinated centres was very important, as the mannose derivatives $\mathbf{3}$ and $\mathbf{4}$ displayed much lower affinity. The 1,2-dideoxy derivative (not shown) was an even poorer binder. This work led DiMagno to propose the 'polar hydrophobicity' concept, in which he synthesised the hexafluorinated pyranose 5,12, 13 and established its much faster GLUT-1 mediated erythrocyte membrane transport rate compared to glucose. Later, O'Hagan synthesised the corresponding 2,3,4-trideoxy-2,3,4trifluoroglucose derivative 6 and showed that its transport rate is slightly slower than that of glucose. ${ }^{14}$ Other syntheses of 6 have been reported. ${ }^{15,16}$ Recently, Giguere synthesised a large number of 2,3,4-trideoxy-2,3,4-trifluorinated sugars, including the fluorinated galactose derivative 7 . Its $\beta$-thionaphthyl glycoside displayed weak ( $\mathrm{IC}_{50}$ 34-38 $\left.\mu \mathrm{M}\right)$ antiproliferative activity against a panel of cancer cell lines. ${ }^{17}$

\footnotetext{
a. School of Chemistry, University of Southampton, Highfield, Southampton SO171BJ, UK.

+ These authors contributed equally to this manuscript.

tElectronic Supplementary Information (ESI) available: Copies of NMR spectra of the novel compounds, crystallographic details for compounds 15a, 16d, and 19. See DOI: $10.1039 / \times 0 \times x 00000 x$. The NMR FID data is available from the Southampton repository at $\mathrm{DOI}: \mathrm{XXXX}$
}

Our group has synthesised vicinal tetrafluorinated sugar derivativatives including 2,3-dideoxy-2,2,3,3-tetrafluoro-Dthreo-hexopyranose $\mathbf{8}, 18,19$ and showed that its furanosyl-UDP derivative 9 was a superior binder to UGM (galactose mutase) than the native Galp-UDP and Galf-UDP substrates. ${ }^{20,21}$ Examples of vicinally dideoxy-difluorinated sugar derivatives include the 3,4-dideoxy-3,4-difluoro-D-glucopyranose $10^{22}$ and its $-N$-acetyl glucosamine analogue $11,{ }^{23}$ as well as the $N$-acetylgalactosamine $\quad \mathbf{1 2}^{23}$ and 2,3-dideoxy-2,3-difluoro-Dglucopyranose $13 .{ }^{24}$

In addition, our group has developed methodology for fluorosugar lipophilicity measurement, which demonstrated the significant lipophilicity $(\log P)$ increases arising from sugar deoxyfluorinations. ${ }^{25}$ Apart from the number of deoxygenations<smiles>OCC1O[C@H](F)C(F)[C@H](O)[C@H]1O[Te]</smiles><smiles>OCC1O[C@H](O)CCC1F</smiles>
5<smiles>OCC1O[C@H](O)CC(O)C1O</smiles>
$\mathrm{OH}$<smiles>OC1C(F)OC(F)C(F)C1O</smiles><smiles>OCC1O[C@H](O)C(F)C(F)C1F</smiles>

6<smiles></smiles>

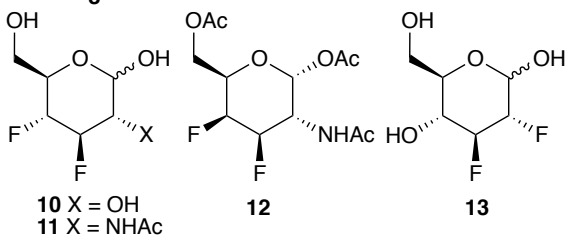

Figure 1. 
and deoxyfluorinations, lipophilicity changes depend on fluorination position and relative stereochemistry, a point which has been further nicely illustrated by Giguere with a large number of the aforementioned 2,3,4-trideoxy-2,3,4trifluorinated sugar analogues. ${ }^{15}$

In galactose, the vicinal dideoxy-difluoro motif has only been described at the 1- and 2-positions. It is synthesised through reaction of tri-O-acetyl or tri-O-benzyl protected D-galactal with $\mathrm{CF}_{3} \mathrm{OF}, \mathrm{XeF}_{2}$, or selectFluor, leading to $14 \mathrm{~b}^{26-30}$ and $14 \mathrm{c}$ (as the $\alpha$-anomer). ${ }^{31} \quad$ Deprotected 1,2-dideoxy-1,2difluorogalactopyranose $14 a$ has also been described (as $\alpha$ anomer). ${ }^{27}$ Here we describe the synthesis of the two other vicinal difluorinated galactose derivatives $\mathbf{1 5}$ and $\mathbf{1 6}$ (Figure 2), both as free hemiacetal and as suitably protected building blocks, eg for activation as glycosyl donor. For 16, the corresponding furanose isomer with required protection at the 5 and 6-positions was also obtained (17b).<smiles>OCC1OC(F)C(F)C(O)C1O</smiles>
14a $\mathrm{R}=\mathrm{H}$ $14 \mathrm{~b} R=A c$ $14 \mathrm{c} R=\mathrm{Bn}$
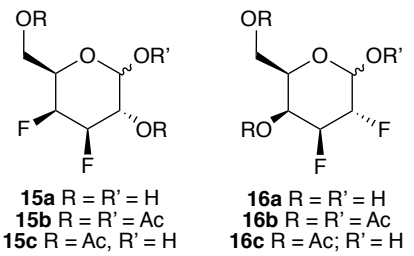

$\begin{aligned} 16 a R & =R^{\prime}=H \\ 16 b R=R^{\prime} & =A c\end{aligned}$ $16 c R=A c ; R^{\prime}=H$

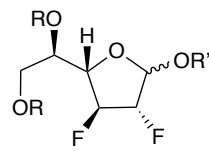

$\left(17 a R=R^{\prime}=H\right)$ $17 b R=R^{\prime}=A c$

Figure 2. Vicinal dideoxy-difluorinated galactoses.

\section{Results and discussion}

The synthesis of 3,4-dideoxy-3,4-difluorogalactose 15a was envisaged from the known fluoro-epoxide $18,{ }^{32}$ which is accessible in two steps from the commercially available "Cerny epoxide" (1,6:3,4-dianhydro-2-O-tosyl- $\beta$-D-galactopyranose, not shown). ${ }^{33}$

Fluoride-mediated epoxide opening of 18 , which as expected occurred regioselectively at the 3-position following the FürstPlattner rule (chair-like transition state), ${ }^{34}$ was slow and required execution in a sealed tube due to the volatility of the starting material. Presumably the electron withdrawing effects of the $4 \beta$-fluoro group and the anomeric acetal hampered the

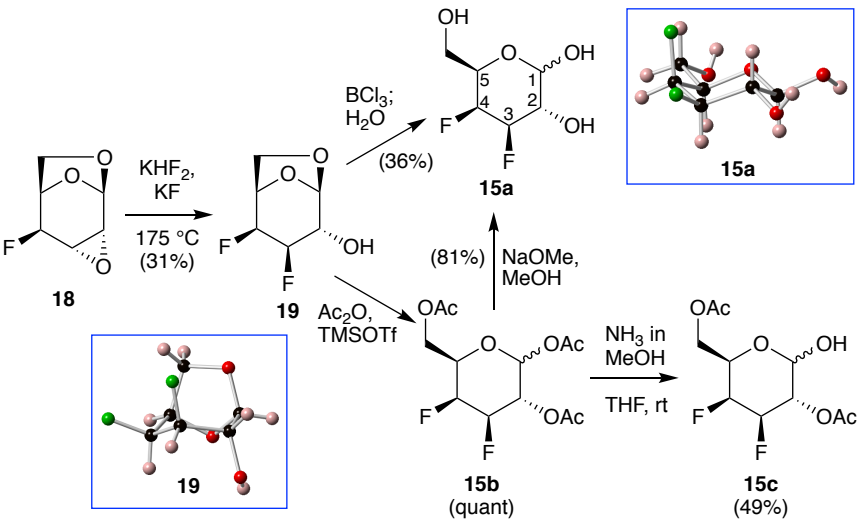

Scheme 1. Synthesis of 3,4-dideoxy-3,4-difluorogalactose (CCDC 15a 1879612; CCDC 191879613. fluoride attack. ${ }^{35-39}$ No product resulting from competing fluoride opening at $\mathrm{C} 2$ was isolated. The axial disposition of the 3-F group in 19 was evident from the large $\mathrm{e}^{40} \mathrm{~J}_{\mathrm{F} 3-\mathrm{H} 4}$ value of 24 $\mathrm{Hz}$, and the diaxial disposition of the newly formed $\mathrm{C2}-\mathrm{C} 3$ fluorohydrin by the smaller ${ }^{3} \mathrm{~J}_{\mathrm{F} 3-\mathrm{H} 2}$ value of $10 \mathrm{~Hz}$ and the large ${ }^{39}$ ${ }^{2} J_{\mathrm{F} 3-\mathrm{C} 2}$ value of $24 \mathrm{~Hz}$. Moreover, 19 proved to be a crystalline solid and X-ray crystallographic analysis confirmed structural assignment (see ESI for full details). Next, opening of the 1,6anhydro bridge was achieved by $\mathrm{BCl}_{3}$ to form the corresponding glycosyl chloride, which was directly hydrolysed to give 15a, albeit in low yield. A much higher-yielding procedure involved TMSOTf-catalysed acetolysis ${ }^{41}$ to give $\mathbf{1 5 b}$, which could then be deprotected to give 15 a in $81 \%$ over 2 steps. Alternatively, selective anomeric deacetylation was achieved to give $15 \mathrm{c}$ in $49 \%$ yield. Pleasingly, a crystal structure of the fully deprotected 15a could be obtained, nicely showing a ${ }^{4} C_{1}$ chair conformation. NMR analysis of 15a in solution (acetone- $d_{6}$ ) shows that the ${ }^{4} C_{1}$ chair conformation is retained: $\mathrm{H} 2$ and $\mathrm{H} 3$ display a $9.6 \mathrm{~Hz}$ coupling constant, indicating a $\sim 180^{\circ}$ dihedral angle and a 2.9 $\mathrm{Hz}$ value is observed for coupling between $\mathrm{H} 3$ and $\mathrm{H} 4$, corresponding to a $\sim 60^{\circ}$ dihedral angle. The coupling constants of $4 \mathrm{~F}$ with $\mathrm{H} 3$ and $\mathrm{H} 5$, both measuring about $28 \mathrm{~Hz}$, as well as these of $\mathrm{F} 3$ with $\mathrm{H} 2$ and $\mathrm{H} 4$, respectively 16 and $7 \mathrm{~Hz}$, show their respective axial and equatorial dispositions. The vicinal $\mathrm{C}-\mathrm{F}$ coupling constants are also diagnostic: ${ }^{3} \mathrm{~J}_{\mathrm{C} 2-\mathrm{F} 4}$ is a small $2.4 \mathrm{~Hz}$, while ${ }^{3} J_{\mathrm{C} 1-\mathrm{F} 3}$ and ${ }^{3} J_{\mathrm{C} 5-\mathrm{F} 3}$ are much larger $(5.3$ and $11.4 \mathrm{~Hz}$ ), which is consistent with an axial F4 and equatorial F3. The corresponding ${ }^{3} J_{\mathrm{C} 2-\mathrm{F} 4}$ value for the glucose derivative $\mathbf{1 0}$ is a much larger $7.7 \mathrm{~Hz}$ (equatorial F4). ${ }^{22}$ Interestingly, the larger $3 J_{\mathrm{H} 2-\mathrm{H} 3}$ value of the difluorogalactose $15 \mathrm{a}(9.6 \mathrm{~Hz}$ for the $\beta$ anomer, acetone- $d_{6}$ ) compared to that of the difluoroglucose 10a $\left(8.8 \mathrm{~Hz} \text { for the } \beta \text {-anomer, acetone- } d_{6}\right)^{22}$ is consistent with the " $\beta$-effect" as proposed by Altona and Haasnoot, ${ }^{42}$ in which the $3 J_{\mathrm{H}-\mathrm{H}}$ value between two antiperiplanar hydrogens is larger when one of these hydrogens itself is antiperiplanar with an electron withdrawing group (as is the case with F4 in 15a). Crich et al. have shown that this ${ }^{3} \mathrm{H}_{\mathrm{H} 2 \text { - } \mathrm{H} 3}$ coupling constant difference is consistent for a large set of glucose/galacto configured derivatives. ${ }^{43}$

The 2,3-dideoxy-2,3-difluorinated galactopyranose 16a and its peracetylated derivative $16 \mathbf{b}$ could be accessed via two different ways from the difluorinated 1,6-anhydro galactosan derivative $\mathbf{2 0}$ (Scheme 2), which was synthesised in 11 steps from levoglucosan according to Sarda et al. ${ }^{15,44}$ At first, it was found that the 1,6 anhydro bridge on $\mathbf{2 0}$ could be cleaved under acetolysis conditions to give the peracetylated 2,3difluorogalactopyranse $\mathbf{1 6} \mathbf{b}$ in excellent yield, although a long reaction time is required ( $64 \mathrm{~h}$ ) due to the electron withdrawing effect of the fluorine atoms. Subsequent acetate hydrolysis using $\mathrm{LiOH}$ proceeded cleanly to afford the free sugar 16a. Alternatively, the reducing sugar 16a could be directly obtained from 20 using $\mathrm{BCl}_{3}$-mediated opening, followed by hydrolysis of the anomeric chloride, which, in contrast to the anhydro bridgeopening of 19 (cf Scheme 1), now proceeded in excellent yield in a much shorter reaction time ( $2 \mathrm{~h}$ ). Finally, selective anomeric 
deprotection could be achieved using methanolic ammonia in $\mathrm{THF}$, leading to $16 \mathrm{c}$ in $84 \%$ yield.

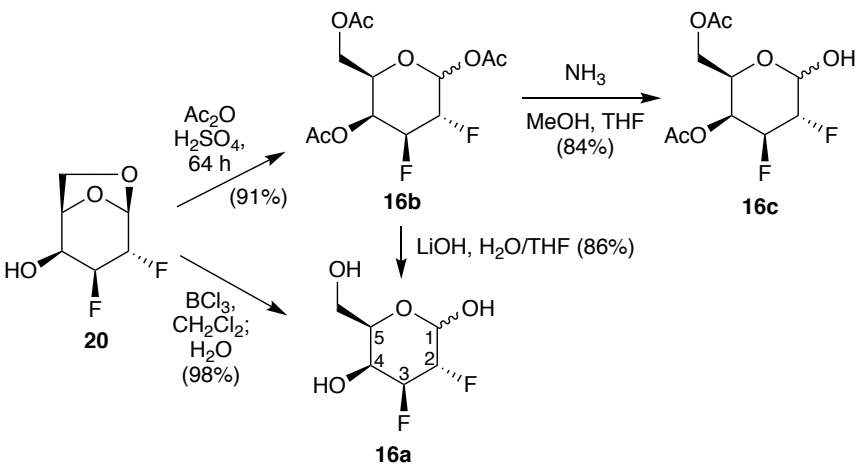

Scheme 2. Synthesis of 2,3-dideoxy-2,3-difluorogalactose 16.

NMR analysis of $16 a\left(D_{2} \mathrm{O}\right)$ showed the occurrence of a ${ }^{4} C_{1}$ conformation. The vicinal $\mathrm{H} 2-\mathrm{H} 3$ coupling constant of $9.1-9.5 \mathrm{~Hz}$ indicates an antiperiplanar position, and the equatorial positions of both fluorine atoms are also evident from the large ${ }^{3} J_{\mathrm{C} 4-\mathrm{F} 2}(8.0-8.6 \mathrm{~Hz})$ and ${ }^{3} J_{\mathrm{C} 1-\mathrm{F} 3} /{ }^{3} J_{\mathrm{C} 5-\mathrm{F} 3}(10-11$ and $6.0-6.7 \mathrm{~Hz})$ values. The smaller magnitude of the $3 \mathrm{~J}_{\mathrm{H} 2 \text { - } \mathrm{H} 3}$ coupling constant for the 2,3-difluorinated glucose derivative $\mathbf{1 3}^{24}\left(\mathrm{D}_{2} \mathrm{O}, 8.8 \mathrm{~Hz}\right.$ for both anomers) is also consistent with the Haasnoot/Altona $\beta$ effect.

The synthesis of the corresponding protected furanose isomer 17b was investigated starting from the pyranose derivatives. Many excellent pyranose-to-furanose isomerisation protocols have been reported, ${ }^{45-50}$ which unfortunately are not all applicable starting from 16a due to the presence of the vicinal fluorine motif at $\mathrm{C} 2$ and $\mathrm{C} 3$.

We first envisaged to obtain the furanose $17 \mathbf{b}$ following an acetylation/isomerisation precedent with 2-deoxy-2fluorogalactose $\mathbf{2 1}$ from Liu and co-workers (Scheme 3). ${ }^{51}$ When applied from 16a however, this led to the almost exclusive formation of the peracetylated pyranose $\mathbf{1 6 b}$, with only trace amounts of furanose product $\mathbf{1 7 b}$ observed by ${ }^{1} \mathrm{H}$ and ${ }^{19} \mathrm{~F}$ NMR analysis.

A different protecting group approach towards the furanose form was inspired by the work of Hricovíniová et al., which showed that treatment of gulose 24 (featuring an axial 4-OH group) with dimethoxypropane in glyme in the presence of $\mathrm{CaSO}_{4}$ led to the formation of the gulofuranose acetal 25 in 66\% yield. ${ }^{52}$ Although formation of the corresponding pyranose is not specifically mentioned in the paper, it is described in the experimental procedure that the formation of another product was detected by TLC analysis. In the case of 16a, even though the absence of hydroxyl groups at $\mathrm{C} 2 / \mathrm{C} 3$ does not allow formation of a stable [3.3.0] fused bicyclic ring (such as in 25), it was assumed that the $6-\mathrm{OH}$ group of 16 a would react first, resulting in the preferential formation of the 5,6-acetonide group under kinetic conditions.

Hence, 16a was treated under the Hricovíniová conditions (A). After $16 \mathrm{~h}$, analysis by TLC indicated the formation of two products, with a very distinct retention factor, which after separation by column chromatography were identified as the pyranose derivative $16 \mathrm{c}$ as the major product, and the desired galactofuranose 17c as minor product. Optimisation was achieved by using similar kinetic acetal formation conditions that our group had developed for the selective protection of arabitol: 53 the reaction temperature was increased to allow faster dissolution of $\mathbf{1 6 a}$, an increased catalyst loading was used to accelerate the reaction, and neutralisation of the acid catalyst after reaction was carried out at the elevated temperature. Indeed, reaction was now complete in a muchreduced reaction time, giving the furanose $17 \mathrm{c}$ as the major product in $44 \%$ yield next to the pyranose derivative $16 \mathrm{~d}$ in 32 $\%$ yield. Interestingly, when treated in the same reaction conditions, it was found that isomerisation of the pyranose acetal $16 \mathbf{d}$ can be initiated, to reach a roughly 1:1 mixture of ring isomers of $\mathbf{1 6 d}$ and $\mathbf{1 7 c}$.

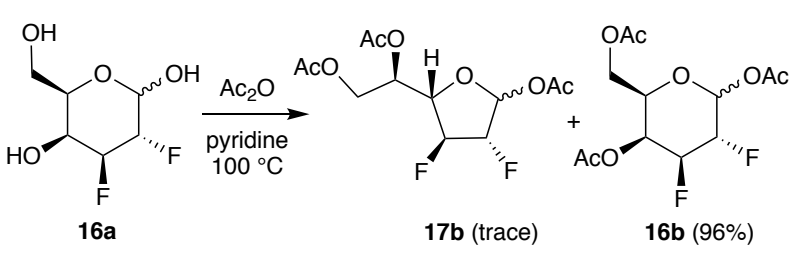

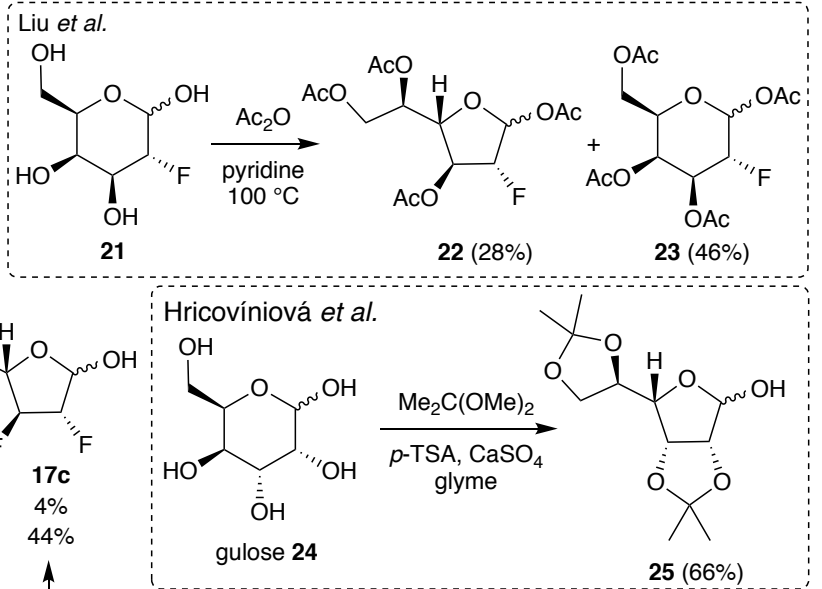

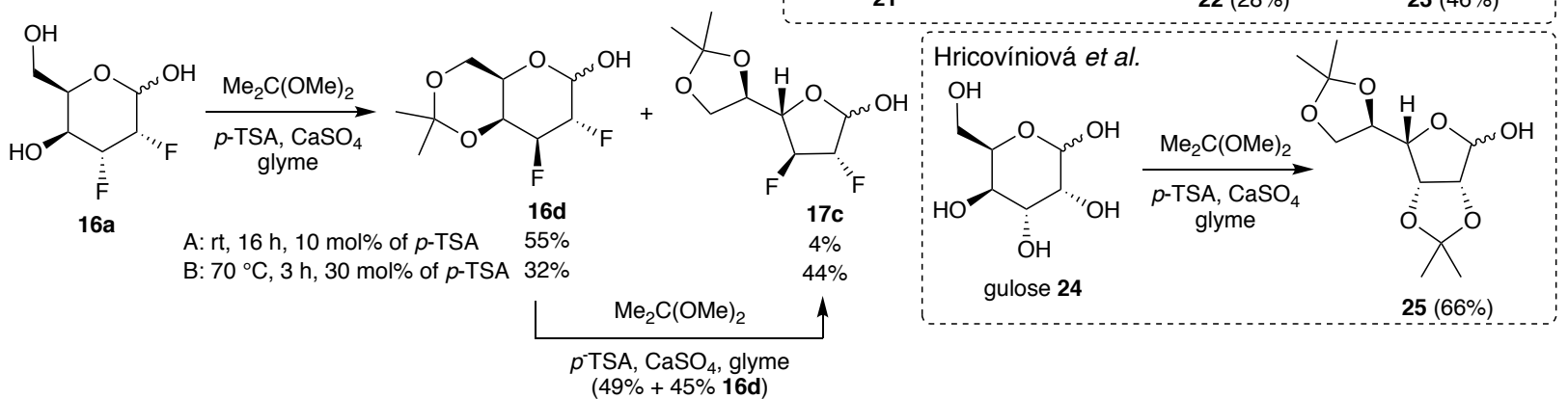

Scheme 3. Synthesis of the galactofuranose 17. 


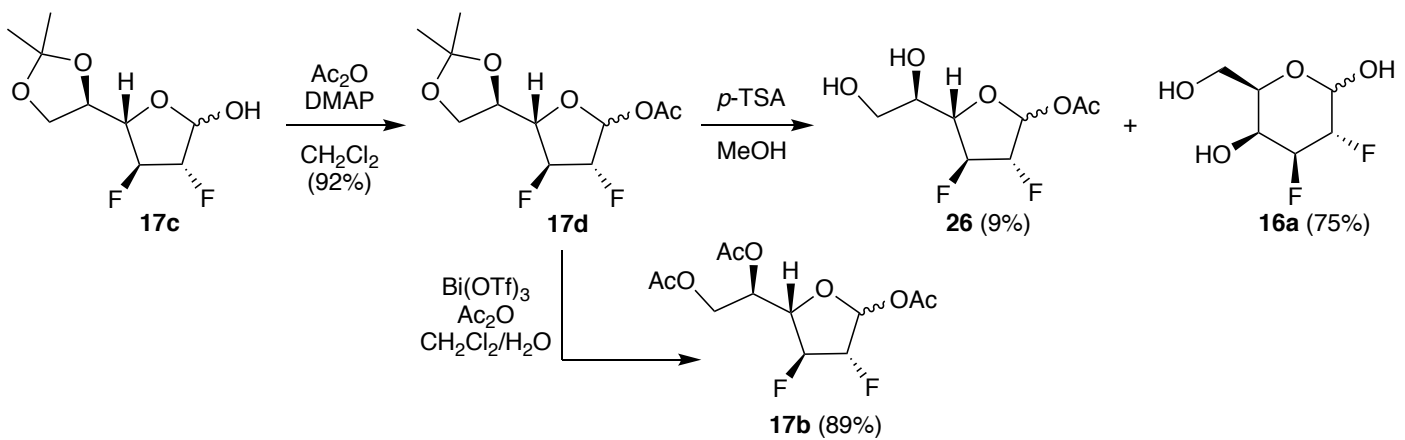

Scheme 4. Protecting group switch to obtain tri-O-acetyl-2,3-dideoxy-2,3-difluorofuranose 17b.

Both products could be assigned by NMR analysis. Thus, in the case of the furanose derivative, a correlation point can be observed in the $\mathrm{HMBC}$ spectrum between $\mathrm{H}-1$ and $\mathrm{C}-4$ (for the major anomer), indicative of a 5-membered sugar ring. Conversely, in the case of the pyranose, a correlation point can be observed in the HMBC spectrum between $\mathrm{H}-1$ and $\mathrm{C}-5$ (for the major anomer), confirming the 6-membered ring composition. In addition, $\mathbf{1 6 d}$ proved crystalline, and single crystal X-ray analysis confirmed its structure (Figure 3 ).

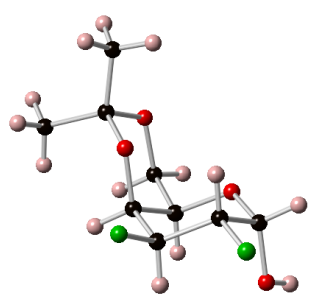

Figure 3. Crystal structure of the pyranose acetonide 16d (CCDC 1455286).

Next, an acetonide to acetyl protecting group swap, in order to allow a wider range of glycosylation conditions, was attempted. To avoid ring isomerisation to the more stable pyranose form, the anomeric hemiacetal group was first converted to the corresponding acetate 17d (Scheme 4). Unfortunately, subsequent removal of the acetonide protecting group using $p$ TSA was accompanied by isomerisation to the pyranose 16a instead of the desired furanose $\mathbf{2 6}$. Treatment of $\mathbf{1 7 d}$ with aqueous $\mathrm{AcOH}$ at $55^{\circ} \mathrm{C}$ for $2 \mathrm{~h}$ led to recovery of the starting material (not shown). However, the target furanose 17b was successfully synthesized from $\mathbf{1 7 d}$ using one-pot acetonide-todiacetate procedure as described by Zhang et al. ${ }^{54}$ This involves reaction with bismuth triflate hydrate as Lewis-acid with 3.8 equiv of $\mathrm{Ac}_{2} \mathrm{O}$ in dichloromethane, and was reported to be suitable for similar protecting group switch on hexofuranoses. Interestingly, this reaction was initially tried with anhydrous $\mathrm{Bi}(\mathrm{OTf})_{3}$, but full conversion was only achieved by adding a few drops of water to the reaction mixture. Pleasingly, under these conditions, diol acetylation after acetonide deprotection is faster than furanose-to-pyranose rearrangement, leading to the desired 2,3-dideoxy-2,3-difluorogalactofuranosyl donor $\mathbf{1 7 b}$ in excellent yield.

\section{Conclusions}

The synthesis of three novel vicinal dideoxy-difluorinated galactose sugar derrivatives is described. 3,4-Dideoxy-3,4difluoro-D-galactopyranose is synthesised in 6 or 7 steps from levoglucosan in per-acetylated or fully deprotected form. The first fluorination step is a retentive deoxyfluorination reaction as reported by the group of Karban, ${ }^{32}$ and the second fluorination is a regioselective epoxide opening. The synthesis of 2,3-dideoxy-2,3-difluoro-D-galactopyranose is based on work by Sarda, 44 and it was shown that a pyranose-into-furanose isomerisation protocol based on acetonide formation allowed access to the corresponding furanose form in good yields. A key finding was that replacement of the acetonide at $\mathrm{OH} 5 / \mathrm{OH} 6$ by a more stable acetate protecting group (for glycosylation purposes) could be achieved without re-isomerisation side reaction back to the pyranose form, using a bismuth triflate catalysed reaction. Both unprotected difluorinated galactopyranose derivatives were shown to exist in the ${ }^{4} C_{1}$ conformation, and NMR-based analysis allows to conclude that this conformation is retained in the solution phase.

\section{Experimental section}

Chemical reagents were obtained from commercial sources and used without further purification, unless stated otherwise. All air/moisture sensitive reactions were carried out under inert atmosphere (Ar) in flame-dried glassware. Anhydrous bottles of THF (tetrahydrofuran), toluene, $\mathrm{CH}_{2} \mathrm{Cl}_{2}$ and $\mathrm{Et}_{3} \mathrm{~N}$, bought from commercial sources, were used for the reactions. When appropriate, other reagents and solvents were purified by standard techniques.

Reactions were monitored by TLC (MERCK Kieselgel 60 F254, aluminium sheet), visualised under UV light ( $254 \mathrm{~nm})$, and by staining with $\mathrm{KMnO}_{4}(10 \%$ aq. $)$ or vanillin. Column chromatography was performed on silica gel (MERCK Geduran $60 \AA$, particle size $40-63 \mu \mathrm{m}$ ). All reported solvent mixtures are volume measures. Normal phase preparative HPLC was carried out using Biorad Bio-Sil D 90-10 columns $(250 \times 10 \mathrm{~mm}$ at 10 $\mathrm{mL} \cdot \mathrm{min}^{-1}$ and $1250 \times 22 \mathrm{~mm}$ at $\left.20 \mathrm{~mL} \mathrm{~min}-1\right)$. Reverse phase 
preparative HPLC was carried out using a Waters Xselect C18 column $\left(19 \times 250 \mathrm{~mm}\right.$ at $\left.17 \mathrm{~mL} \cdot \mathrm{min}^{-1}\right)$. Size exclusion chromatography was carried out on a GE healthcare column (XK16 model), packed with Sephadex LH-20 (MeOH used as mobile phase). The column was connected to an AKTA apparatus (1-5 $\left.\mathrm{mL} \cdot \mathrm{min}^{-1}\right)$.

${ }^{1} \mathrm{H},{ }^{13} \mathrm{C}$, and ${ }^{19} \mathrm{~F}$ spectra were recorded in $\mathrm{CDCl}_{3}$, acetone- $d_{6}$, methanol- $d_{4}$ or $\mathrm{D}_{2} \mathrm{O}$ using a BRUKER AV400 $(400,101,376$ and $162 \mathrm{MHz}$ respectively) and $\operatorname{AV} 500(500,125,470$ and $202 \mathrm{MHz}$ respectively) spectrometers. ${ }^{1} \mathrm{H}$ and ${ }^{13} \mathrm{C}$ chemical shifts $(\delta)$ are quoted in ppm relative to residual solvent peaks as appropriate. ${ }^{19} \mathrm{~F}$ spectra were externally referenced to $\mathrm{CFCl}_{3}$. The coupling constants $(J)$ were recorded in Hertz $(\mathrm{Hz})$. The coupling constants have not been averaged. Fourier-transform infrared (FT-IR) spectra are reported in wavenumbers $\left(\mathrm{cm}^{-1}\right)$ and were recorded as neat films on a Thermo Scientific Nicolet iS5 spectrometer using neat samples (solid or liquid). Electrospray mass spectra were obtained from a Waters 2700 sample manager ESI, and recorded in $\mathrm{m} / \mathrm{z}$ (abundance). HRMS was obtained from a Bruker APEX III FT-ICR-MS. Samples were run in HPLC methanol or MeCN. Optical rotations were recorded on an Optical Activity POLAAR 2001 at 589 nm.

\section{1,6-Anhydro-3,4-dideoxy-3,4-difluoro- $\beta$-D-galactopyranose 19}

To a solution of 1,6:2,3-dianhydro-4-deoxy-4-fluoro- $\beta$-Dgulopyranose $18^{32}$ (447 mg, $3.1 \mathrm{mmol}$ ) in ethylene glycol ( $7 \mathrm{~mL}$ ) in a sealed tube and under argon, was added KF (1.09 g, 18.8 $\mathrm{mmol})$ and $\mathrm{KHF}_{2}(1.40 \mathrm{~g}, 17.9 \mathrm{mmol})$. The mixture was stirred at $175^{\circ} \mathrm{C}$. After $15 \mathrm{~h}$ the solution was cooled to $\mathrm{rt}$ and a solution of sat. aq. $\mathrm{NaHCO}_{3}(7 \mathrm{~mL})$ was added dropwise, followed by extraction with $\mathrm{Et}_{2} \mathrm{O}(5 \times 70 \mathrm{~mL})$. The combined organic layers were dried over $\mathrm{MgSO}_{4}$, filtered, concentrated in vacuo and then purified by column chromatography (silica, petroleum ether/ $\left.\mathrm{Et}_{2} \mathrm{O}, 1: 1\right)$ to give 19 (155 mg, $\left.0.9 \mathrm{mmol}, 31 \%\right)$ as a white fluffy solid, and recovered starting material 18 (20 mg, 0.14 $\mathrm{mmol}, 4 \%)$. $\mathbf{R}_{\mathrm{f}} 0.40$ (hexane/acetone, 1:1); $\mathbf{m p} 134 \stackrel{\circ}{\circ} \mathrm{C} ;[\boldsymbol{\alpha}]_{\mathrm{D}}{ }^{22}$ 19.6 (c 1, $\mathrm{CHCl}_{3}$ ); IR 3442 (br), 2958 (br), 2922 (br), 1479 (m), 1065 (s), 1038 (s) 1026 (s), 966 (s), 697 (s) cm ${ }^{-1}$; ${ }^{1}$ H NMR (500 $\mathrm{MHz}_{\mathrm{CDCl}}$ ): $\delta 5.42(1 \mathrm{H}, \mathrm{dt}, J 5.3,1.5 \mathrm{~Hz}, \mathrm{H}-1), 4.88(1 \mathrm{H}, \mathrm{m}, J 49.9$ $\mathrm{Hz}$ was observed, $\mathrm{H}-3$ ), 4.85 (1H, ddtdd, J 44.3, 24.6, 4.2, 1.3, 0.5 $\mathrm{Hz}, \mathrm{H}-4), 4.65(1 \mathrm{H}$, br. d, J $4.6 \mathrm{~Hz}, \mathrm{H}-5), 4.37(1 \mathrm{H}, \mathrm{br}$ dd, J 7.8, 0.8 $\mathrm{Hz}, \mathrm{H}-6 e n d o), 4.06-3.97(1 \mathrm{H}, \mathrm{m}, \mathrm{H}-2), 3.79$ (1H, ddtt J 7.8, 5.0, 1.3, $0.6 \mathrm{~Hz}, \mathrm{H}-6 \mathrm{exo}), 1.96(1 \mathrm{H}, \mathrm{d}, J 8.9 \mathrm{~Hz}, \mathrm{OH}) \mathrm{ppm} ;{ }^{1} \mathbf{H}\left\{{ }^{19} \mathrm{~F}\right\}$ NMR

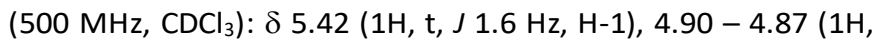
$\mathrm{m}, \mathrm{H}-3), 4.84(1 \mathrm{H}, \mathrm{brtdd}, \mathrm{J} 4.2,1.3,0.5 \mathrm{~Hz}, \mathrm{H}-4), 4.65(1 \mathrm{H}, \mathrm{tdd}, \mathrm{J}$ 5.0, 0.9, 0.4 Hz, H-5), 4.37 (1H, dt, J 7.8, 0.6 Hz, H-6endo), 4.02 $(1 \mathrm{H}, \mathrm{dt}, J$ 8.9, $1.9 \mathrm{~Hz}, \mathrm{H}-2), 3.79(1 \mathrm{H}, \mathrm{dddd}, J$ 7.8, 5.1, 1.2, $0.6 \mathrm{~Hz}$, H-6exo), $1.96(1 \mathrm{H}, \mathrm{d}, J 8.9 \mathrm{~Hz}, \mathrm{OH})$ ppm; ${ }^{13} \mathrm{C}$ NMR $(126 \mathrm{MHz}$, $\mathrm{CDCl}_{3}$ ): $\delta 100.7$ (C-1), 87.5 (dd, J 188.1, $14.3 \mathrm{~Hz}, \mathrm{C}-4$ ), 82.7 (dd, J 194.2, 16.1 Hz, C-3), 72.0 (dd, J 27.4, 1.0 Hz, C-5), 70.7 (dd, J 23.8, $2.9 \mathrm{~Hz}, \mathrm{C}-2), 64.1$ (dd, J4.3, $1.7 \mathrm{~Hz}, \mathrm{C}-6)$ ppm; ${ }^{19} \mathrm{~F}$ NMR $(471 \mathrm{MHz}$, $\mathrm{CDCl}_{3}$ ): $\delta$-203.9 (1F, ddddt, J 49.2, 24.9, 11.3, 4.8, $\left.1.1 \mathrm{~Hz}, \mathrm{~F}-3\right)$, 208.5 - -208.7 (1F, m, J $44.4 \mathrm{~Hz}$ can be observed, F-4) ppm; ${ }^{19} \mathrm{~F}\left\{{ }^{1} \mathrm{H}\right\}$ NMR $\left(471 \mathrm{MHz}, \mathrm{CDCl}_{3}\right): \delta-203.9(1 \mathrm{~F}, \mathrm{~d}, J 5.0 \mathrm{~Hz}, \mathrm{~F}-3),-$ 208.6 (1F, d, J $5.0 \mathrm{~Hz}$, F-4) ppm; HRMS (ESI-) for $\mathrm{C}_{6} \mathrm{H}_{7} \mathrm{~F}_{2} \mathrm{O}_{3}$ calcd. 165.0363 , found 165.0369 .

\section{3,4-Dideoxy-3,4-difluoro-D-galactopyranose 15a (from 19)}

To a solution of 19 (100 mg, $0.602 \mathrm{mmol})$ in $\mathrm{CH}_{2} \mathrm{Cl}_{2}(6.9 \mathrm{~mL})$ at 0 ${ }^{\circ} \mathrm{C}$ was added $\mathrm{BCl}_{3}\left(1 \mathrm{M}\right.$ in $\left.\mathrm{CH}_{2} \mathrm{Cl}_{2}, 2.39 \mathrm{~mL}, 2.39 \mathrm{mmol}\right)$. The reaction was then allowed to reach rt. After $3 \mathrm{~h}$ sat. aq. $\mathrm{NaHCO}_{3}$ $\left(20 \mathrm{~mL}\right.$ ) was added at $0{ }^{\circ} \mathrm{C}$. After $30 \mathrm{~min}$ the reaction was concentrated. The crude residue was then purified by column chromatography (silica, petroleum ether/acetone, 1:1) to give $\mathbf{1 5 a}$ as a white solid ( $40 \mathrm{mg}, 0.22 \mathrm{~mol}, 36 \%$ ). $\mathbf{R}_{\mathrm{f}} 0.20$ (petroleum ether/acetone, 1:1), $\mathbf{R}_{\mathbf{f}} 0.23$ (1:1 acetone:hexane); $\mathbf{m p} 170-171$

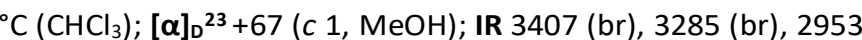
(w), 1140 (s), 1079 (s), 1106 (s), 1004 (s), 799 (s), 695 (s) cm-1; ${ }^{1} \mathrm{H}$ NMR $\left(500 \mathrm{MHz}\right.$, acetone- $\left.d_{6}\right)$ : (ratio $\left.\alpha: \beta 2: 1\right) \delta 5.96(1 \mathrm{H}, \mathrm{dd}, J$ 6.9, $1.1 \mathrm{~Hz}, \mathrm{OH}-1 \beta), 5.80(1 \mathrm{H}, \mathrm{dd}, J 4.1,0.9 \mathrm{~Hz}, \mathrm{OH}-1 \alpha), 5.23(1 \mathrm{H}$, app. q, J $4.2 \mathrm{~Hz}, \mathrm{H}-1 \alpha), 5.06(1 \mathrm{H}, \mathrm{ddd}, J 52.0,8.0,2.8 \mathrm{~Hz}, \mathrm{H}-4 \alpha$ ), $4.99(1 \mathrm{H}$, ddd, J 51.6, 6.9, $3.0 \mathrm{~Hz}, \mathrm{H}-4 \beta), 4.76(1 \mathrm{H}$, dddd, J 48.1, 27.7, 9.8, $2.9 \mathrm{~Hz}, \mathrm{H}-3 \alpha), 4.65(1 \mathrm{H}, \mathrm{d}, J 4.4 \mathrm{~Hz}, \mathrm{OH}-2 \beta), 4.56(1 \mathrm{H}$, app. br t, J 7.7, $6.9 \mathrm{~Hz}, \mathrm{H}-1 \beta), 4.58$ (2H, dddd, J 46.9, 27.9, 9.6,

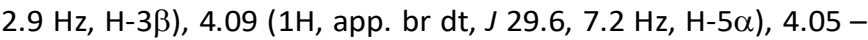
$4.02(2 \mathrm{H}, \mathrm{m}, \mathrm{OH}-2 \alpha+\mathrm{OH}-6 \beta), 3.96(1 \mathrm{H}, \mathrm{dd}, J 6.6,5.0 \mathrm{~Hz}, \mathrm{OH}-6 \alpha)$, $3.94-3.90(1 \mathrm{H}, \mathrm{m}, \mathrm{H}-2 \alpha), 3.75-3.60(5 \mathrm{H}, \mathrm{m}, \mathrm{H}-2 \alpha+2 \times \mathrm{H}-6 \alpha$ $+2 \times \mathrm{H}-6 \beta) \mathrm{ppm} ;{ }^{13} \mathrm{C}$ NMR $\left(126 \mathrm{MHz}\right.$, acetone- $\left.d_{6}\right)$ : (ratio $\left.\alpha: \beta 2: 1\right)$ $\delta 97.4$ (d, J $11.4 \mathrm{~Hz}, \mathrm{C}-1 \beta$ ), 93.7 (d, J $10.5 \mathrm{~Hz}, \mathrm{C}-1 \alpha$ ), 92.3 (dd, J $187.8,17.6 \mathrm{~Hz}, \mathrm{C}-3 \beta$ ), 90.2 (dd, J 186.6, $17.6 \mathrm{~Hz}, \mathrm{C}-3 \alpha$ ), 87.9 (dd,

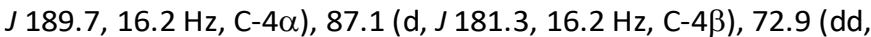

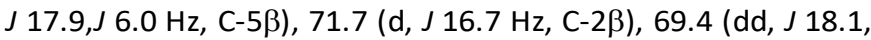
$5.3 \mathrm{~Hz}, \mathrm{C}-5 \alpha), 68.2(\mathrm{dd}, J 17.6,2.4 \mathrm{~Hz}, \mathrm{C}-2 \alpha), 60.0-59.8(\mathrm{~m}, \mathrm{C}-$ $6 \alpha+\mathrm{C} 6 \beta)$ ppm; ${ }^{19} \mathrm{~F}$ NMR $\left(471 \mathrm{MHz}\right.$, acetone- $\left.d_{6}\right)$ : (ratio $\left.\alpha: \beta 2: 1\right) \delta$ -201.3 (1F, app dtd, J 47.0, 16.3, 6.8 Hz, F-3ß), -206.1 - -206.3

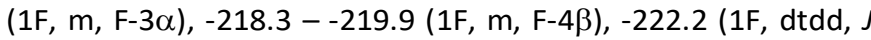
51.9, 28.3, 15.5, $0.9 \mathrm{~Hz}, \mathrm{~F}-4 \alpha) \mathrm{ppm} ;{ }^{19} \mathrm{~F}\left\{{ }^{1} \mathrm{H}\right\}$ NMR $(471 \mathrm{MHz}$, acetone- $\left.d_{6}\right)$ : (ratio $\left.\alpha: \beta 2: 1\right) \delta-201.3(1 \mathrm{~F}, \mathrm{~d}, J 16.3 \mathrm{~Hz}, \mathrm{~F}-3 \beta)$, $206.2(1 F, d, J 15.5 \mathrm{~Hz}, \mathrm{~F}-3 \alpha),-219.0$ (1F, d, J $16.3 \mathrm{~Hz}, \mathrm{~F}-4 \beta)$, $222.2(1 \mathrm{~F}, \mathrm{~d}, J 15.5 \mathrm{~Hz}, \mathrm{~F}-4 \alpha)$ ppm; HRMS (ESI+) for $\mathrm{C}_{6} \mathrm{H}_{10} \mathrm{~F}_{2} \mathrm{NaO}_{4}$ calcd. 207.0444, found 207.0440.

\section{1,2,6-Tri-O-acetyl-3,4-dideoxy-3,4-difluoro-D-galactopyranose 15b.}

To a solution of 19 (300 mg, $1.81 \mathrm{mmol})$ in $\mathrm{Ac}_{2} \mathrm{O}(2.7 \mathrm{~mL})$ at $0{ }^{\circ} \mathrm{C}$ was added TMSOTf $(0.07 \mathrm{~mL}, 0.36 \mathrm{mmol})$. After $30 \mathrm{~min}$ the reaction mixture was warmed up to $\mathrm{rt}$. After an additional $2 \mathrm{~h}$ the mixture was diluted with $\mathrm{CH}_{2} \mathrm{Cl}_{2}(5 \mathrm{~mL})$ and then slowly neutralised with a solution of sat. aq. $\mathrm{NaHCO}_{3}$. The aqueous phase was then separated and extracted with $\mathrm{CH}_{2} \mathrm{Cl}_{2}(3 \times 20 \mathrm{~mL})$, the combined organic phases were washed with $\mathrm{NaHCO}_{3}(3 \times 20$ $\mathrm{mL}), \mathrm{H}_{2} \mathrm{O}(20 \mathrm{~mL})$, dried over $\mathrm{MgSO}_{4}$, filtered, concentrated in vacuo and purified by column chromatography (silica, hexane/acetone, $7: 3$ ) to give $\mathbf{1 5 b}$ as a mixture of anomers (ratio $\alpha: \beta 2: 15$ ), as a pale yellow oil (560 mg, $1.81 \mathrm{mmol}$, quant.). $\mathbf{R}_{\mathbf{f}}$ 0.29 (hexane/acetone, 7:3); IR 1750 (s), 1372 (m), 1229 (s), 1214 (s), 1077 (s) cm ${ }^{-1} ;{ }^{1} \mathrm{H}$ NMR (500 MHz, CDCl $): \delta 6.41(1 \mathrm{H}, \mathrm{t}, J 4.3$ $\mathrm{Hz}, \mathrm{H}-1 \alpha), 5.65(1 \mathrm{H}, \mathrm{d}, J 8.0 \mathrm{~Hz}, \mathrm{H}-1 \beta), 5.46$ (1H, app. tdd, J 10.5, 3.8, $0.9 \mathrm{~Hz}, \mathrm{H}-2 \alpha), 5.06(1 \mathrm{H}$, ddd, J 50.5, 7.6, $2.6 \mathrm{~Hz}, \mathrm{H}-4 \alpha), 4.99$

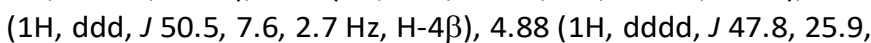

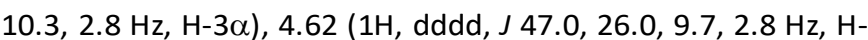

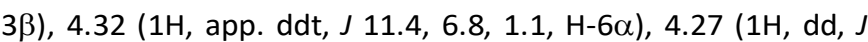
$\left.11.4,6.4, H-6^{\prime} \alpha\right), 4.17(1 \mathrm{H}, \mathrm{br}, \mathrm{dt}$, J 27.7, $6.6 \mathrm{~Hz}, \mathrm{H}-5 \alpha), 3.89(1 \mathrm{H}$, 
dtd, J 25.3, 6.5, $1.3 \mathrm{~Hz}, \mathrm{H}-5 \beta$ ), $2.15\left(3 \mathrm{H}, \mathrm{s}, \mathrm{COCH}_{3}-\alpha\right), 2.14(3 \mathrm{H}, \mathrm{s}$, $\left.\mathrm{COCH}_{3}-\beta\right), 2.12\left(3 \mathrm{H}, \mathrm{s}, \mathrm{COCH}_{3}-\beta\right), 2.10\left(3 \mathrm{H}, \mathrm{s}, \mathrm{COCH}_{3}-\beta\right), 2.10(3 \mathrm{H}$, $\left.\mathrm{s}, \mathrm{COCH}_{3}-\alpha\right), 2.09\left(3 \mathrm{H}, \mathrm{s}, \mathrm{COCH}_{3}-\alpha\right) \mathrm{ppm} ;{ }^{13} \mathrm{C}$ NMR $(126 \mathrm{MHz}$, $\mathrm{CDCl}_{3}$ ): $\delta 170.6$ (s, $\left.\underline{\mathrm{COCH}}_{3}-\alpha\right), 170.5$ (s, $\left.\underline{\mathrm{COCH}}_{3}-\beta\right), 169.7$ (s, $\mathrm{COCH}_{3}-\alpha$ ), 169.20 (s, $\left.\mathrm{COCH}_{3}-\beta\right), 169.19$ (s, $\left.\mathrm{COCH}_{3}-\beta\right), 168.7$ (s,

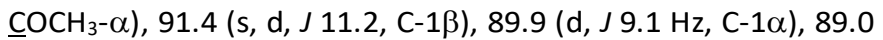
(dd, J 195.7, $18.1 \mathrm{~Hz}, \mathrm{C}-3 \beta$ ), 86.5 (dd, J 187.4, $16.7 \mathrm{~Hz}, \mathrm{C}-4 \alpha$ ),

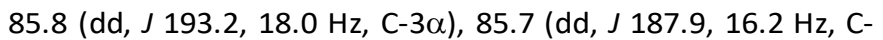

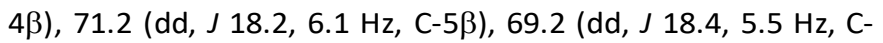

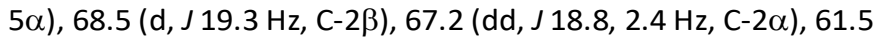

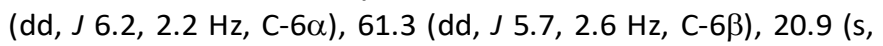

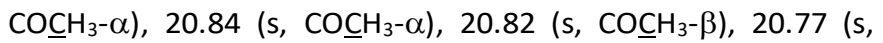
$\left.\mathrm{CO}_{\mathrm{C}} \mathrm{H}_{3}-\beta\right), 20.6$ (s, $\left.\mathrm{CO}_{\mathrm{C}} \mathrm{H}_{3}-\alpha\right) \mathrm{ppm}$. One carbon resonance for the minor $\beta$-anomer could not be located; ${ }^{19} \mathrm{~F}$ NMR $(471 \mathrm{MHz}$, $\left.\mathrm{CDCl}_{3}\right): \delta-201.3--201.6(1 \mathrm{~F}, \mathrm{~m}, J 46.9 \mathrm{~Hz}$ is observed, $\mathrm{F}-3 \beta)$, $205.0--205.3(1 \mathrm{~F}, \mathrm{~m}, J 47.7 \mathrm{~Hz}$, is observed, $\mathrm{F}-3 \alpha),-217.8(1 \mathrm{~F}$, $\mathrm{dtd}, J$ 50.3, 25.6, $14.9 \mathrm{~Hz}, \mathrm{~F}-4 \beta),-220.0$ (1F, dtdd, J 50.6, 27.4, 14.9, 0.9 Hz, F-4 $\alpha$ ) ppm; ${ }^{19} \mathrm{~F}\left\{{ }^{1} \mathrm{H}\right\}$ NMR $\left(471 \mathrm{MHz}, \mathrm{CDCl}_{3}\right): \delta-201.3$

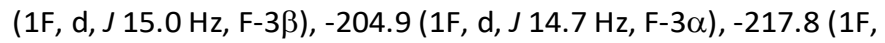
d, J $15.0 \mathrm{~Hz}, \mathrm{~F}-4 \beta$ ), -220.0 (1F, d, J $15.0 \mathrm{~Hz}, \mathrm{~F}-4 \alpha)$ ppm; MS (ESI+): $333[\mathrm{M}+\mathrm{Na}]^{+}$; HRMS (ESI+) for $\mathrm{C}_{12} \mathrm{H}_{16} \mathrm{~F}_{2} \mathrm{NaO}_{7}$ calcd. 333.0761, found 333.0761 .

\section{3,4-Dideoxy-3,4-difluoro-D-galactopyranose 15a (from 15b)}

To a solution of $15 \mathrm{~b}(200 \mathrm{mg}, 0.64 \mathrm{mmol})$ in $\mathrm{MeOH}$ was added MeONa (25\% wt, $0.03 \mathrm{~mL}, 0.15 \mathrm{mmol})$. After $3 \mathrm{~h}$ the reaction was neutralised to $\mathrm{pH} 7$ using Amberlite ${ }^{\circledR}$ IR120 hydrogen form resin, filtered, and concentrated. The crude residue was purified by column chromatography ( $50 \%$ acetone in hexane) to give first the partially deprotected 6-O-acetyl product $(14 \mathrm{mg}, 0.06$ $\mathrm{mmol}, 10 \%$ ) and then the fully deprotected product 15a (96 mg, $0.52 \mathrm{mmol}, 81 \%)$ as a white solid.

\section{2,6-Di-O-acetyl-3,4-dideoxy-3,4-difluoro-D-galactopyranose 15c}

To a solution of $\mathbf{1 5 b}$ (352 mg, $1.13 \mathrm{mmol})$ in THF (14 mL) at $0{ }^{\circ} \mathrm{C}$ was added a solution of $\mathrm{NH}_{3}$ in $\mathrm{MeOH}(7 \mathrm{~N}, 3.17 \mathrm{~mL})$. After $1 \mathrm{~h}$ the reaction mixture was allowed to warm to $\mathrm{rt}$, and stirred for $3 \mathrm{~h}$. The reaction mixture was concentrated in vacuo and purified by column chromatography (silica, petroleum ether/acetone, $7 / 3$ ) to give title compound $\mathbf{1 5 c}$ as a pale yellow oil (150 mg, $0.56 \mathrm{mmol}, 49 \%)$. $\mathbf{R}_{\mathrm{f}} 0.29$ (hexane/acetone, 6:4);

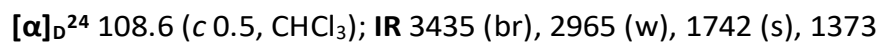
(m), 1229 (s), 1048 (s) cm ${ }^{-1}$; ${ }^{1} \mathbf{H}$ NMR ( $\alpha$-anomer only) (400 MHz, $\mathrm{CDCl}_{3}$ ): (ratio $\alpha: \beta$ 5:1) $\delta 5.56(1 \mathrm{H}$, app. q, J $3.8 \mathrm{~Hz}, \mathrm{H}-1 \alpha), 5.26$ (1H, app. td, J 10.4, 3.6 Hz, H-2 $\alpha$ ), 5.03 (1H, ddd, J 51.0, 7.6, 2.9 $\mathrm{Hz}, \mathrm{H}-4 \alpha), 4.95$ (1H, dddd, J 48.2, 26.2, 10.3, $2.8 \mathrm{~Hz}, \mathrm{H}-3 \alpha), 4.40$ $-4.27(3 \mathrm{H}, \mathrm{m}, \mathrm{H}-5 \alpha, \mathrm{H}-6 \alpha), 3.10(1 \mathrm{H}, \mathrm{d}, \mathrm{J} 2.7 \mathrm{~Hz}, \mathrm{OH} \alpha), 2.17(3 \mathrm{H}$, $\left.\mathrm{s}, \mathrm{COC}_{\underline{3}}\right), 2.11\left(3 \mathrm{H}, \mathrm{s}, \mathrm{COC}_{\underline{3}}\right)$ ppm; ${ }^{13} \mathrm{C}$ NMR ( $\alpha$-anomer only) (101 MHz, $\left.\mathrm{CDCl}_{3}\right)$ : (ratio $\alpha: \beta$ 5:1) $\delta 170.6\left(\mathrm{COOCH}_{3}\right), 170.2$ $\left(\mathrm{COOCH}_{3}\right), 90.9$ (d, J $\left.8.8 \mathrm{~Hz}, \mathrm{C}-1 \alpha\right), 86.9$ (dd, J 186.3, $16.9 \mathrm{~Hz}, \mathrm{C}-$

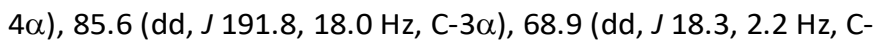
$2 \alpha), 66.6(\mathrm{dd}, J$ 18.0, $5.5 \mathrm{~Hz}, \mathrm{C}-5 \alpha), 61.8(\mathrm{dd}, J 5.9,2.2 \mathrm{~Hz}, \mathrm{C}-6 \alpha)$, $20.8\left(\mathrm{COOCH}_{3}\right), 20.7\left(\mathrm{COOCH}_{3}\right)$ ppm; ${ }^{19} \mathrm{~F}$ NMR $\left(376 \mathrm{MHz}, \mathrm{CDCl}_{3}\right)$ : (ratio $\alpha: \beta 5: 1) \delta-201.4(1 \mathrm{~F}, \mathrm{~m}, J 46.8 \mathrm{~Hz}$ is observed, $\mathrm{F}-3 \beta)$, $206.2(1 F, m, J 48.5 \mathrm{~Hz}$ is observed, $\mathrm{F}-3 \alpha$ ), -217.8 (1F, dtd, J 50.3,

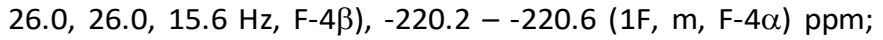

${ }^{19} \mathrm{~F}\left\{{ }^{1} \mathrm{H}\right\}$ NMR $\left(376 \mathrm{MHz}, \mathrm{CDCl}_{3}\right)$ : (ratio $\left.\alpha: \beta 5: 1\right) \delta-201.5(1 \mathrm{~F}, \mathrm{~d}, J$ $15.6 \mathrm{~Hz}, \mathrm{~F}-3 \beta),-206.3(1 \mathrm{~F}, \mathrm{~d}, J 15.6 \mathrm{~Hz}, \mathrm{~F}-3 \alpha),-217.9(1 \mathrm{~F}, \mathrm{~d}, J 15.6$ $\mathrm{Hz}, \mathrm{F}-4 \beta),-220.5$ (1F, d, J $15.6 \mathrm{~Hz}, \mathrm{~F}-4 \alpha$ ) ppm; HRMS (ESI+) for $\mathrm{C}_{10} \mathrm{H}_{14} \mathrm{~F}_{2} \mathrm{NaO}_{6}$ calcd. 291.0656, found 291.0651.

\section{1,4,6-Tri-O-acetyl-2,3-dideoxy-2,3-difluorogalactose $16 \mathrm{~b}$}

To a solution of $20(627 \mathrm{mg}, 3.77 \mathrm{mmol})$ in $\mathrm{Ac}_{2} \mathrm{O}(14 \mathrm{~mL}, 148$ $\mathrm{mmol}$ ) at $0{ }^{\circ} \mathrm{C}$ was added dropwise concentrated sulfuric acid (1 $\mathrm{mL}, 18.6 \mathrm{mmol})$. After $64 \mathrm{~h}$ at $\mathrm{rt}$ the reaction was neutralised at $0{ }^{\circ} \mathrm{C}$ with a sat. aq. solution of $\mathrm{NaHCO}_{3}(40 \mathrm{~mL})$. The mixture was extracted with EtOAc $(40 \mathrm{~mL}$, then $2 \times 30 \mathrm{~mL})$, and the combined organic phases were dried over $\mathrm{MgSO}_{4}$, filtered, concentrated in vacuo and purified by column chromatography (silica, petroleum ether/acetone $75: 25$ ) to give $\mathbf{1 6 b}$ as a white solid (1.06 g, 91\%, ratio $\alpha: \beta$ 4:1). $\mathbf{R}_{\mathbf{f}} 0.35$ (hexane/acetone, 7:3); IR (neat): 1751 (s), 1214 (s), 1082 (m), 1041 (w), 1014 (w) cm ${ }^{-1} ;{ }^{1} \mathbf{H}$ NMR (500 MHz, CDCl $)$ : $\delta 6.46(1 \mathrm{H}$, app. t, J $4.2 \mathrm{~Hz}, \mathrm{H}-1 \alpha), 5.73$ $(1 \mathrm{H}, \mathrm{dd}, J 8.0,4.1 \mathrm{~Hz}, \mathrm{H}-1 \beta), 5.70-5.64(1 \mathrm{H}, \mathrm{m}, \mathrm{H}-4 \alpha)$, $5.61(1 \mathrm{H}$, dddd, J 5.2, 3.9, 2.6, $1.1 \mathrm{~Hz}, \mathrm{H}-4 \beta), 5.10-4.88(2 \mathrm{H}, \mathrm{m}, \mathrm{H}-2 \alpha, \mathrm{H}-$ $3 \alpha), 4.87-4.64(2 \mathrm{H}, \mathrm{m}, \mathrm{H}-2 \beta, \mathrm{H}-3 \beta), 4.25(1 \mathrm{H}, \mathrm{tt}, J 6.7,1.2 \mathrm{~Hz}$, $\mathrm{H}-5 \alpha), 4.16(1 \mathrm{H}, \mathrm{dd}, J 11.4,6.4 \mathrm{~Hz}, \mathrm{H}-6 \beta), 4.12(1 \mathrm{H}, \mathrm{dd}, J$ 11.4, $6.6 \mathrm{~Hz}, \mathrm{H}-6 \alpha), 4.12-4.06\left(1 \mathrm{H}, \mathrm{m}, \mathrm{H}-6^{\prime} \beta\right), 4.06(1 \mathrm{H}, \mathrm{ddd}, J 11.3$, 6.8, $\left.1.1 \mathrm{~Hz}, \mathrm{H}-6^{\prime} \alpha\right), 3.99$ ( $1 \mathrm{H}, \mathrm{tdd}$, J 6.5, 1.7, $1.3 \mathrm{~Hz}, \mathrm{H}-5 \beta$ ), 2.19 $\left(3 \mathrm{H}, \mathrm{s}, \mathrm{COCH}_{3}-\beta\right), 2.17\left(3 \mathrm{H}, \mathrm{s}, \mathrm{COC}_{3}-\alpha\right), 2.16\left(3 \mathrm{H}, \mathrm{s}, \mathrm{COCH}_{3}-\beta\right)$, $2.16\left(3 \mathrm{H}, \mathrm{s}, \mathrm{COC}_{3}-\alpha\right), 2.05\left(3 \mathrm{H}, \mathrm{s}, \mathrm{COCH}_{3}-\beta\right), 2.05\left(3 \mathrm{H}, \mathrm{s}, \mathrm{COC}_{3}{ }^{-}\right.$

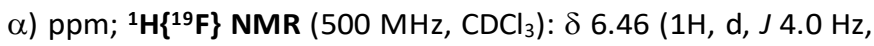
$\mathrm{H}-1 \alpha), 5.73(1 \mathrm{H}, \mathrm{d}, J 7.8 \mathrm{~Hz}, \mathrm{H}-1 \beta), 5.67(1 \mathrm{H}, \mathrm{dd}, J 3.7,1.3 \mathrm{~Hz}, \mathrm{H}-$ $4 \alpha), 5.61(1 \mathrm{H}, \mathrm{dd}, J 3.9,1.1 \mathrm{~Hz}, \mathrm{H}-4 \beta), 5.02(1 \mathrm{H}, \mathrm{dd}, J 9.4,3.8 \mathrm{~Hz}$, $\mathrm{H}-3 \alpha), 4.96(1 \mathrm{H}, \mathrm{dd}, J$ 9.5, $4.2 \mathrm{~Hz}, \mathrm{H}-2 \alpha), 4.79(1 \mathrm{H}, \mathrm{dd}, J$ 9.1, 4.0 $\mathrm{Hz}, \mathrm{H}-3 \beta), 4.73(1 \mathrm{H}, \mathrm{dd}, J 9.1,7.9 \mathrm{~Hz}, \mathrm{H}-2 \beta), 4.25(1 \mathrm{H}, \mathrm{td}, J 6.7$, $1.3 \mathrm{~Hz}, \mathrm{H}-5 \alpha), 4.16(1 \mathrm{H}, \mathrm{dd}, J 11.4,6.4 \mathrm{~Hz}, \mathrm{H}-6 \beta), 4.12(1 \mathrm{H}, \mathrm{dd}, J$ 11.4, $6.6 \mathrm{~Hz}, \mathrm{H}-6 \alpha), 4.12-4.06\left(1 \mathrm{H}, \mathrm{dd}, J 11.5,6.6 \mathrm{~Hz}, \mathrm{H}-6^{\prime} \beta\right)$, $4.06\left(1 \mathrm{H}, \mathrm{dd}, J 11.4,6.7 \mathrm{~Hz}, \mathrm{H}-6^{\prime} \alpha\right), 3.99(1 \mathrm{H}, \mathrm{td}, J 6.5,1.2 \mathrm{~Hz}, \mathrm{H}-$ $5 \beta), 2.19\left(3 \mathrm{H}, \mathrm{s}, \mathrm{COC}_{3}-\beta\right), 2.17\left(3 \mathrm{H}, \mathrm{s}, \mathrm{COC}_{3}-\beta\right), 2.16(3 \mathrm{H}, \mathrm{s}$, $\left.\mathrm{COC}_{3}-\beta\right), 2.16\left(3 \mathrm{H}, \mathrm{s}, \mathrm{COC} \underline{H}_{3}-\beta\right), 2.05\left(3 \mathrm{H}, \mathrm{s}, \mathrm{COCH}_{3}-\beta\right), 2.05(3 \mathrm{H}$, s, $\left.\mathrm{COCH}_{3}-\beta\right)$ ppm; ${ }^{13} \mathrm{C}$ NMR (126 MHz, $\left.\mathrm{CDCl}_{3}\right): \delta 170.3\left(\mathrm{COCH}_{3}-\right.$ $\alpha+\beta), 169.58\left(\underline{\mathrm{COCH}}_{3}-\alpha\right), 169.55\left(\underline{\mathrm{COCH}}_{3}-\beta\right), 168.8\left(\underline{\mathrm{COCH}}_{3}-\beta\right)$, $168.6\left(\mathrm{COCH}_{3}-\alpha\right), 90.8(\mathrm{dd}, J 24.6,11.4 \mathrm{~Hz}, \mathrm{C}-1 \beta), 89.1$ (dd, J 22.7 , $9.5 \mathrm{~Hz}, \mathrm{C}-1 \alpha), 88.7\left(\mathrm{dd}, J 194.4,19.1 \mathrm{~Hz}, \mathrm{C}_{2} \beta\right.$ or $\left.\mathrm{C}_{3} \beta\right), 87.8(\mathrm{dd}$, $J 188.5,20.5 \mathrm{~Hz}, \mathrm{C}_{2} \beta$ or $\left.\mathrm{C}_{3} \beta\right), 86.2(\mathrm{dd}, J 192.2,19.1 \mathrm{~Hz}, \mathrm{C}-2 \alpha)$,

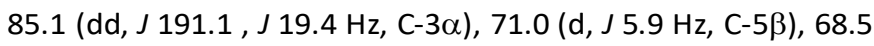

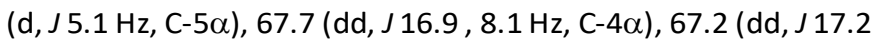
, $8.4 \mathrm{~Hz}, \mathrm{C}-4 \beta$ ), 60.9 (d, J $2.2 \mathrm{~Hz}, \mathrm{C}-6 \alpha), 60.8$ (d, J $2.2 \mathrm{~Hz}, \mathrm{C}-6 \beta)$, $20.7\left(\underline{\mathrm{COCH}}_{30}-\alpha+\beta\right), 20.56\left(\mathrm{COCH}_{3}-\alpha+\beta\right), 20.45\left(\underline{\mathrm{COCH}}_{3}-\alpha+\beta\right)$ ppm; ${ }^{19} \mathrm{~F}$ NMR (471 MHz, $\left.\mathrm{CDCl}_{3}\right): \delta-200.8(1 \mathrm{~F}, \mathrm{~m}, \mathrm{~F} \beta),-204.8--$ 205.1 (1F, $\mathrm{m}, J 48.6 \mathrm{~Hz}$ is observed, $\mathrm{F} \alpha$ ), -208.2 (1F, dtdd, J 51.9, 13.9, 4.3, $2.5 \mathrm{~Hz}, \mathrm{~F} \beta),-209.6--209.9(1 \mathrm{~F}, \mathrm{~m}, \mathrm{~F} \alpha) \mathrm{ppm} ;{ }^{19} \mathrm{~F}\left\{{ }^{1} \mathrm{H}\right\}$ NMR (471 MHz, CDCl $\left.{ }_{3}\right): \delta-200.8(1 F, d, J 13.9 \mathrm{~Hz}, \mathrm{~F} \beta),-205.0(1 \mathrm{~F}$, d, J $13.6 \mathrm{~Hz}, \mathrm{F \alpha}),-208.2(1 \mathrm{~F}, \mathrm{~d}, J 13.9 \mathrm{~Hz}, \mathrm{~F} \beta),-209.8(1 \mathrm{~F}, \mathrm{~d}, J 13.6$ $\mathrm{Hz}, \mathrm{F} \alpha)$ ppm; MS (ESI+) $333[\mathrm{M}+\mathrm{Na}]^{+}$; HRMS (ESI+) for $\mathrm{C}_{12} \mathrm{H}_{16} \mathrm{~F}_{2} \mathrm{NaO}_{7}[\mathrm{M}+\mathrm{Na}]^{+}$cacld 333.0761, found 333.0754.

\section{2,3-Dideoxy-2,3-difluorogalactose 16a (from 20)}

To a solution of 20 (3.10 g, $18.7 \mathrm{mmol})$ in $\mathrm{CH}_{2} \mathrm{Cl}_{2}$ at $0{ }^{\circ} \mathrm{C}$ was added dropwise $\mathrm{BCl}_{3}\left(1 \mathrm{M}\right.$ in $\mathrm{CH}_{2} \mathrm{Cl}_{2}, 37.3 \mathrm{~mL}, 37.3 \mathrm{mmol}$ ), over a period of $30 \mathrm{~min}$. The mixture was stirred at $0{ }^{\circ} \mathrm{C}$ for $30 \mathrm{~min}$, 
allowed to warm up to rt and stirred for $3.5 \mathrm{~h}$. Water $(100 \mathrm{~mL})$ was added, and the resulting biphasic mixture was stirred for 5$10 \mathrm{~min}$, before concentrating in vacuo and purification by column chromatography (silica, $\mathrm{CH}_{2} \mathrm{Cl}_{2} / \mathrm{MeOH}$, 9:1) to afford $16 \mathrm{a}$ as a colourless oil (3.38 g, $18.4 \mathrm{mmol}, 98 \%)$.

\section{2,3-Dideoxy-2,3-difluorogalactose 16a (from 16b)}

To a solution of $16 \mathrm{~b}(1.06 \mathrm{~g}, 3.42 \mathrm{mmol}$ ) in a mixture of THF (30 $\mathrm{mL}$ ) and $\mathrm{H}_{2} \mathrm{O}(2.5 \mathrm{~mL})$ at $\mathrm{rt}$ was added $\mathrm{LiOH}(370 \mathrm{mg}, 15.5 \mathrm{mmol})$. The reaction was stirred for $1 \mathrm{~h}$, during which an emulsion was observed. The solvent was concentrated in vacuo and purified by column chromatography (silica, $\mathrm{CH}_{2} \mathrm{Cl}_{2} / \mathrm{MeOH}, 90: 10$ ) to give 16a as a colourless oil (540 mg, $2.93 \mathrm{mmol}, 86 \%$ ). $\mathbf{R}_{\mathbf{f}} 0.11$ (hexane/acetone, 1:1); [ $\boldsymbol{\alpha}]_{D^{20}}+81.4$ (c 1, MeOH); IR (neat) 3379 (m br), 2917 (w), $2496(\mathrm{~m}), 1029$ (s) cm ${ }^{-1}$; ${ }^{1} \mathbf{H}$ NMR $(500 \mathrm{MHz}$, $\mathrm{D}_{2} \mathrm{O}$ ): (ratio $\left.\alpha: \beta 1: 1\right) \delta 5.51(1 \mathrm{H}$, br. t, J $4.3 \mathrm{~Hz}, \mathrm{H}-1 \alpha), 5.10-4.77$ (4H, m, H-1 $\beta, \mathrm{H}-2 \alpha, \mathrm{H}-3 \alpha, \mathrm{H}-2 \beta), 4.58$ (1H, dddd, J 52.4, 13.6,

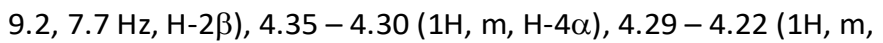
$\mathrm{H}-4 \beta), 4.17-4.09(1 \mathrm{H}, \mathrm{m}, \mathrm{H}-5 \alpha), 3.83-3.69(5 \mathrm{H}, \mathrm{m}, \mathrm{H}-5 \beta, \mathrm{H}-$ $\left.6 \alpha+\beta, H-6^{\prime} \alpha+\beta\right)$ ppm; ${ }^{1} \mathbf{H}\left\{{ }^{19} \mathrm{~F}\right\}$ NMR (ratio $\left.\alpha: \beta 1: 1\right): \delta 5.51(1 \mathrm{H}$, d, J $4.1 \mathrm{~Hz}, \mathrm{H}-1 \alpha), 4.98(1 \mathrm{H}, \mathrm{dd}, J$ 9.6, $3.6 \mathrm{~Hz}, \mathrm{H}-3 \alpha), 4.91(1 \mathrm{H}, \mathrm{dd}$,

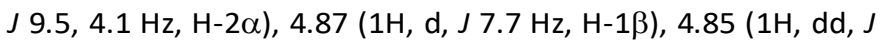
9.1, 3.7 Hz, H-3ß), $4.58(1 \mathrm{H}, \mathrm{dd}, J$ 9.1, $7.7 \mathrm{~Hz}, \mathrm{H}-2 \beta), 4.32(1 \mathrm{H}, \mathrm{dd}$, J 3.6, $1.1 \mathrm{~Hz}, \mathrm{H}-4 \alpha), 4.26(1 \mathrm{H}, \mathrm{dd}, J 3.7,0.9 \mathrm{~Hz}, \mathrm{H}-4 \beta), 4.13(1 \mathrm{H}$, app. td, J 6.2, $1.1 \mathrm{~Hz}, \mathrm{H}-5 \alpha)$, $3.78(1 \mathrm{H}, \mathrm{dd}, J 12.0,8.5 \mathrm{~Hz}, \mathrm{H}-6 \alpha)$, 3.78 - $3.71\left(4 \mathrm{H}, \mathrm{m}, \mathrm{H}-5 \beta, \mathrm{H}-6 \beta, \mathrm{H}-6^{\prime} \alpha+\beta\right) \mathrm{ppm}$; ${ }^{13} \mathrm{C}$ NMR (126 $\mathrm{MHz}, \mathrm{D}_{2} \mathrm{O}$ ): (ratio $\alpha: \beta$ 1:1) $\delta 93.5$ (dd, J 23.2, J $11.1 \mathrm{~Hz}, \mathrm{C}-1 \beta$ ), 91.1 (dd, J 184.7, 17.5 Hz, C-3ß), 90.7 (dd, J 181.7, $18.4 \mathrm{~Hz}, \mathrm{C}-$

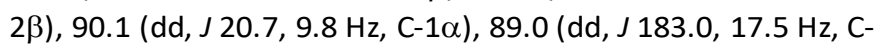

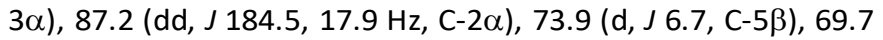

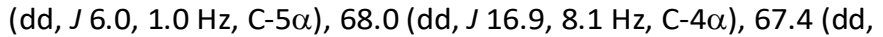

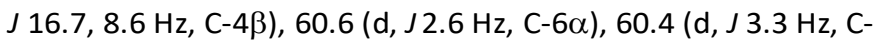

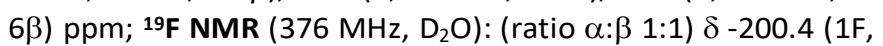

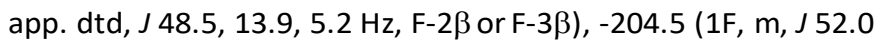
$\mathrm{Hz}$ is observed, $\mathrm{F}-2 \alpha$ or $\mathrm{F}-3 \alpha$ ), $-208.4(1 \mathrm{~F}$, app. dtt, $J$ 52.0, 14.7, $3.5 \mathrm{~Hz}, \mathrm{~F}-2 \beta$ or F-3 $\beta$ ), -208.8 (1F, m, F-2 $\alpha$ or $\mathrm{F}-3 \alpha$ ) ppm; ${ }^{19} \mathrm{~F}\left\{{ }^{1} \mathrm{H}\right\}$ NMR (471 MHz, $\left.D_{2} \mathrm{O}\right)$ : (ratio $\alpha: \beta$ 1:1) $\delta-200.3(1 \mathrm{~F}, \mathrm{~d}, J 14.3 \mathrm{~Hz}$, $\mathrm{F}-2 \beta$ or $\mathrm{F}-3 \beta),-204.4(1 \mathrm{~F}, \mathrm{~d}, J 14.7 \mathrm{~Hz}, \mathrm{~F}-2 \alpha$ or $\mathrm{F}-3 \alpha),-208.3(1 \mathrm{~F}$, d, J $14.3 \mathrm{~Hz}, \mathrm{~F}-2 \beta$ or F-3 $\beta$ ), $-208.6(1 \mathrm{~F}, \mathrm{~d}, J 14.7 \mathrm{~Hz}, \mathrm{~F}-2 \alpha$ or F-3 $\alpha$ ) ppm HRMS (ESI+) for $\mathrm{C}_{6} \mathrm{H}_{10} \mathrm{~F}_{2} \mathrm{NaO}_{4}[\mathrm{M}+\mathrm{Na}]^{+}$calcd. 207.0444, found. 207.0436.

\section{4,6-Di-O-Acetyl-2,3-dideoxy-2,3-difluorogalactose 16c}

To a solution of $16 \mathrm{~b}\left(483 \mathrm{mg}, 1.56 \mathrm{mmol}\right.$ ) in THF at $0{ }^{\circ} \mathrm{C}$ was added a solution of $\mathrm{NH}_{3}$ in $\mathrm{MeOH}(7 \mathrm{M}, 4.5 \mathrm{~mL}, 31 \mathrm{mmol})$. The mixture was stirred at $0{ }^{\circ} \mathrm{C}$ for $1 \mathrm{~h}$, then at $\mathrm{rt}$ for $6 \mathrm{~h}$, before concentrating the solvent in vacuo and purification by column chromatography (silica, petroleum ether/acetone, 7:3 to $6: 4$ ) afforded $16 \mathrm{c}$ as a viscous oil (350 $\mathrm{mg}, 1.30 \mathrm{mmol}, 84 \%)$. $\mathbf{R}_{\boldsymbol{f}} 0.38$ $(\alpha), 0.35(\beta)$ (hexane/acetone, 1:1); $[\alpha]_{D}{ }^{24}+97.3$ (c 1, $\mathrm{CDCl}_{3}$ ); IR (neat) 3427 (m, br.), 1740 (s), 1373 (m), 1223 (s), 1049 (s) cm-1; ${ }^{1} \mathrm{H}$ NMR $\left(500 \mathrm{MHz}, \mathrm{CDCl}_{3}\right.$ ) (ratio $\left.\alpha: \beta 3: 1\right) \delta 5.65(1 \mathrm{H}, \mathrm{br} \mathrm{dtd}, J] \mathrm{z}$, 4.1, $1.3 \mathrm{~Hz}, \mathrm{H}-4 \alpha$ ), 5.59 (1H, dddd, J 5.2, 3.9, 2.6, $1.1 \mathrm{~Hz}, \mathrm{H}-4 \beta$ ), $5.55(1 \mathrm{H}, \mathrm{q}, J 3.7 \mathrm{~Hz}, \mathrm{H}-1 \alpha), 5.08$ ( $1 \mathrm{H}$, dddd, J 49.0, 11.9, 9.4, 3.9 $\mathrm{Hz}, \mathrm{H}-3 \alpha$ ), $4.87-4.83(1 \mathrm{H}, \mathrm{br} \mathrm{m}, \mathrm{H}-1 \beta), 4.84$ (1H, dddd, J 50.7, $11.8,9.4,3.8 \mathrm{~Hz}, \mathrm{H}-3 \alpha$ ), $4.74(1 \mathrm{H}$, dddd, J 47.8, 13.1, 9.0, $3.9 \mathrm{~Hz}$,
$\mathrm{H}-3 \beta), 4.57(1 \mathrm{H}, \mathrm{dddd}, J 51.8,13.1,9.0,7.7 \mathrm{~Hz}, \mathrm{H}-2 \beta), 4.43(1 \mathrm{H}$, $\mathrm{tt}, J$ 6.5, $1.3 \mathrm{~Hz}, \mathrm{H}-5 \alpha$ ), 4.18 (1H, br dd, J 11.3, $6.2 \mathrm{~Hz}, \mathrm{H}-6 \beta), 4.15$ $(1 \mathrm{H}, \mathrm{dd}, J 11.4,6.1 \mathrm{~Hz}, \mathrm{H}-6 \alpha), 4.13(1 \mathrm{H}$, ddd, J 11.7, 6.9, $1.0 \mathrm{~Hz}$, $\left.\mathrm{H}-6 \beta^{\prime}\right), 4.08\left(1 \mathrm{H}, \mathrm{ddd}, J 11.4,6.9,1.0 \mathrm{~Hz}, \mathrm{H}-6 \alpha^{\prime}\right), 3.90(1 \mathrm{H}, \mathrm{tt}, J$

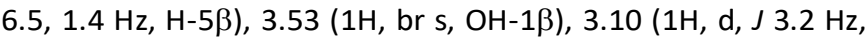
$\mathrm{OH}-1 \alpha), 2.16\left(3 \mathrm{H}, \mathrm{s}, \mathrm{COC}_{\underline{3}}-\beta\right), 2.15\left(3 \mathrm{H}, \mathrm{s}, \mathrm{COC}_{\underline{3}}-\alpha\right), 2.07(6 \mathrm{H}$, br s, $\left.\mathrm{COCH}_{3}-\alpha+\beta\right) ;{ }^{1}{ }^{1}\left\{{ }^{19} \mathrm{~F}\right\}$ NMR $\left(500 \mathrm{MHz}, \mathrm{CDCl}_{3}\right)$ : (ratio $\left.\alpha: \beta 3: 1\right)$ $\delta 5.65(1 \mathrm{H}, \mathrm{dd}, J 3.9,1.3 \mathrm{~Hz}, \mathrm{H}-4 \alpha), 5.59(1 \mathrm{H}, \mathrm{dd}, J 4.0,1.1 \mathrm{~Hz}, \mathrm{H}-$ $4 \beta), 5.55(1 \mathrm{H}, \mathrm{t}, J 3.4 \mathrm{~Hz}, \mathrm{H}-1 \alpha), 5.07(1 \mathrm{H}, \mathrm{dd}, J 9.3,3.9 \mathrm{~Hz}, \mathrm{H}-$ $3 \alpha), 4.84(1 \mathrm{H}, \mathrm{br} d d, J 9.5,3.7 \mathrm{~Hz}, \mathrm{H}-1 \beta+\mathrm{H}-3 \alpha), 4.74(1 \mathrm{H}, \mathrm{dd}, J$ 9.1, 4.0 Hz, H-3ß), $4.57(1 \mathrm{H}, \mathrm{dd}, J 9.1,7.6 \mathrm{~Hz}, \mathrm{H}-2 \beta), 4.43(1 \mathrm{H}$, dddd, J 6.9, 6.1, 1.3, $0.5 \mathrm{~Hz}, \mathrm{H}-5 \alpha), 4.18(1 \mathrm{H}, \mathrm{dd}, J 11.4,6.2 \mathrm{~Hz}$, $\mathrm{H}-6 \beta), 4.15(1 \mathrm{H}, \mathrm{dd}, J 11.3,6.1 \mathrm{~Hz}, \mathrm{H}-6 \alpha), 4.13$ (1H, dd, J 11.5, $\left.6.7 \mathrm{~Hz}, \mathrm{H}-6 \beta^{\prime}\right), 4.08\left(1 \mathrm{H}, \mathrm{dd}, J 11.4,6.9 \mathrm{~Hz}, \mathrm{H}-6 \alpha^{\prime}\right), 3.90(1 \mathrm{H}, \mathrm{td}, J$

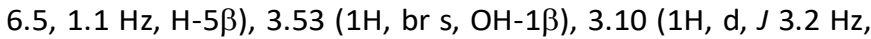
$\mathrm{OH}-1 \alpha), 2.16\left(3 \mathrm{H}, \mathrm{s}, \mathrm{COC}_{\underline{3}}-\beta\right), 2.15\left(3 \mathrm{H}, \mathrm{s}, \mathrm{COC}_{\underline{3}}-\alpha\right), 2.07(6 \mathrm{H}$, br s, $\left.\mathrm{COCH}_{3}-\alpha+\beta\right)$ ppm; ${ }^{13} \mathrm{C}$ NMR $\left(126 \mathrm{MHz}, \mathrm{CDCl}_{3}\right.$ ): (ratio $\alpha: \beta$ $3: 1) \delta 170.7\left(\mathrm{COCH}_{3}-\alpha\right.$ and $\left.\beta\right), 169.94\left(\mathrm{COCH}_{3}-\alpha\right), 169.90$ $\left(\mathrm{COCH}_{3}-\beta\right), 94.5$ (dd, J 23.4, 10.7 Hz, C-1 $\beta$ ), 91.2 (dd, J 21.3, 9.7 $\mathrm{Hz}, \mathrm{C}-1 \alpha$ ), 90.4 (dd, J 186.4, $19.1 \mathrm{~Hz}, \mathrm{C}-2 \beta), 88.9$ (dd, J 193.8, $19.1 \mathrm{~Hz}, \mathrm{C}-3 \beta$ ), 86.8 (dd, J 189.3, $18.6 \mathrm{~Hz}, \mathrm{C}-2 \alpha$ ), 86.2 (dd, J 191.2, $19.1 \mathrm{~Hz}, \mathrm{C}-3 \alpha$ ), 70.5 (d, J $6.4 \mathrm{~Hz}, \mathrm{C}-5 \beta$ ), 68.7 (dd, J 16.9, $8.1 \mathrm{~Hz}, \mathrm{C}-4 \alpha), 67.7$ (dd, J 16.8, 8.5 Hz, C- $4 \beta), 66.6$ (d, J $5.0 \mathrm{~Hz}, \mathrm{C}-$ $5 \alpha), 61.8(d, J 1.7 \mathrm{~Hz}, \mathrm{C}-6 \alpha), 61.6(\mathrm{~d}, J 2.6 \mathrm{~Hz}, \mathrm{C}-6 \beta), 20.86$ $\left(\mathrm{COCH}_{3}-\alpha\right), 20.84\left(\mathrm{COCH}_{3}-\beta\right), 20.72\left(\mathrm{COCH}_{3}-\alpha\right), 20.69\left(\mathrm{COCH}_{3}-\beta\right)$ ppm; ${ }^{19} \mathrm{~F}$ NMR (471 $\mathrm{MHz} \mathrm{CDCl}_{3}$ ) (ratio $\alpha: \beta 3: 1$ ): $\delta-201.0(1 \mathrm{~F}$, app. dtd, J 48.1, 13.6, $5.5 \mathrm{~Hz}, \mathrm{~F}-3 \beta),-206.8(1 \mathrm{~F}, \mathrm{~m}, J 49.3$ can be observed, F-3 $\alpha$ ), -207.0 (1F, app. dtdd, J 52.2 13.9, 3.9, $2.5 \mathrm{~Hz}$, $\mathrm{F}-2 \beta),-208.1$ (1F, dddd, J 50.4, 13.6, 11.8, 3.2 Hz, F-2 $\alpha$ ) ppm; ${ }^{19} \mathrm{~F}\left\{{ }^{1} \mathrm{H}\right\}$ NMR (471 MHz, $\mathrm{CDCl}_{3}$ ) (ratio $\left.\alpha: \beta 3: 1\right): \delta-201.0(1 \mathrm{~F}, \mathrm{~d}, J$

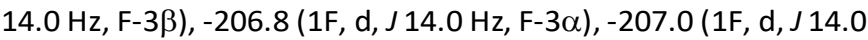
$\mathrm{Hz}, \mathrm{F}-2 \beta),-208.1$ (1F, d, J $14.0 \mathrm{~Hz}, \mathrm{~F}-2 \alpha)$ ppm; MS (ESI+) (m/z) $291[\mathrm{M}+\mathrm{Na}]^{+}$; HRMS (ESI+) for $\mathrm{C}_{10} \mathrm{H}_{14} \mathrm{~F}_{2} \mathrm{NaO}_{6}[\mathrm{M}+\mathrm{Na}]^{+}$calcd. 291.0656, found 291.0646.

\section{5,6-Di-O-isopropylidene-2,3-dideoxy-2,3-difluoro-D- galactofuranose $17 \mathrm{c}$ and 4,6-di- $O$-isopropylidene-2,3-dideoxy-2,3- difluoro-D-galactopyranose 16d (from 16a).}

To a solution of 16 a (636 mg, $3.45 \mathrm{mmol}$ ) in glyme $(42 \mathrm{~mL})$ at $\mathrm{rt}$ was added $\mathrm{CaSO}_{4}$ (dried under vacuum prior to use, $941 \mathrm{mg}$, $6.91 \mathrm{mmol}), 2$,2-dimethoxypropane $(2.14 \mathrm{~mL}, 17.27 \mathrm{mmol})$ and $p$-TSA (177 $\mathrm{mg}, 1.04 \mathrm{mmol}$ ) in this order. The mixture was heated to $70^{\circ} \mathrm{C}$. After $3 \mathrm{~h}$ a sat. aq. solution of $\mathrm{NaHCO}_{3}(20 \mathrm{~mL})$ was added at that temperature. The resulting mixture was filtered over cotton wool, and extracted with EtOAc $(60 \mathrm{~mL}$ then $2 \times 30 \mathrm{~mL}$ ). The organic layers were combined, dried over $\mathrm{MgSO}_{4}$, concentrated in vacuo and then purified by column chromatography (silica, petroleum ether/acetone $85: 15$ to $5: 5$ ) gave first the furanose acetal 17c as a pale yellow oil $(338 \mathrm{mg}$, $1.51 \mathrm{mmol}, 44 \%$ ) and the pyranose acetal $16 \mathrm{~d}$ as a viscous yellow oil ( $283 \mathrm{mg}, 1.10 \mathrm{mmol}, 32 \%$ ).

Data for 2,3-F - -galactopyranose acetal $\mathbf{1 6 d}$ : $\quad \mathbf{R}_{\mathbf{f}} \quad 0.34$ (hexane/acetone, 1:1); [ $\alpha]_{D^{24}}+107.7$ (c 1, $\mathrm{CDCl}_{3}$ ); IR (neat) 3412 (m br), 2995 ( w br), 1385 (m), 1066 (s) cm ${ }^{-1} ;{ }^{1}$ H NMR (500 MHz, $\left.\mathrm{CDCl}_{3}\right)$ : (ratio $\alpha: \beta$ 7:1) $\delta 5.58(1 \mathrm{H}, \mathrm{t}, J 3.95 \mathrm{~Hz}, \mathrm{H}-1 \alpha), 5.11-4.90$ $(2 H, m, H-2 \alpha, H-3 \alpha), 4.81-4.53$ (3H, m, H-1 $\beta, H-2 \beta, H-3 \beta), 4.53$ 
$-4.48(1 \mathrm{H}, \mathrm{m}, \mathrm{H}-4 \alpha), 4.44(1 \mathrm{H}, \mathrm{dddd}, J 5.0,3.8,2.5,1.2 \mathrm{~Hz}, \mathrm{H}-$ $4 \beta), 4.12(1 \mathrm{H}, \mathrm{dd}, J 13.1,1.9 \mathrm{~Hz}, \mathrm{H}-6 \alpha), 4.10(1 \mathrm{H}, \mathrm{dd}, J$ 12.9, 2.1 $\mathrm{Hz}, \mathrm{H}-6 \beta), 3.98\left(1 \mathrm{H}, \mathrm{dt}, J\right.$ 13.0, $\left.1.9 \mathrm{~Hz}, \mathrm{H}-6^{\prime} \beta\right), 3.91(1 \mathrm{H}, \mathrm{dt}, J$ 13.0, $\left.1.9 \mathrm{~Hz}, \mathrm{H}-6^{\prime} \alpha\right), 3.87-3.83(1 \mathrm{H}, \mathrm{m}, \mathrm{H}-5 \alpha), 3.43-3.41(1 \mathrm{H}, \mathrm{m}, \mathrm{H}-$ $5 \beta), 3.29(1 \mathrm{H}, \mathrm{d}, J 2.6 \mathrm{~Hz}, \mathrm{OH}), 1.52-1.46\left(6 \mathrm{H}, \mathrm{m}, \mathrm{C}\left(\mathrm{C}_{3}\right)_{2}-\beta\right)$, $1.50\left(3 \mathrm{H}, \mathrm{s} . \mathrm{C}\left(\mathrm{C}_{3}\right)_{2}-\alpha\right), 1.48\left(3 \mathrm{H}, \mathrm{s}, \mathrm{C}\left(\mathrm{C}_{3}\right)_{2}-\alpha\right) \mathrm{ppm} ;{ }^{13} \mathrm{C}$ NMR $\left(101 \mathrm{MHz}, \mathrm{CDCl}_{3}\right)$ : (ratio $\alpha: \beta$ 7:1) $\delta 99.3\left(\underline{\mathrm{C}}\left(\mathrm{CH}_{3}\right)_{2}-\beta\right), 99.0$ $\left(\underline{\mathrm{C}}\left(\mathrm{CH}_{3}\right)_{2}-\alpha\right), 94.3$ (dd, J 23.4, $\left.10.3 \mathrm{~Hz}, \mathrm{C}-1 \beta\right), 91.4$ (dd, J 21.6, 9.2

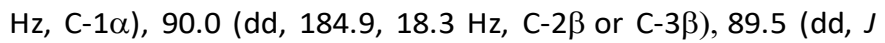
193.7, $18.3 \mathrm{~Hz}, \mathrm{C}-2 \beta$ or C-3 $\beta$ ), 86.51 (dd, J 190.7, $18.3 \mathrm{~Hz}, \mathrm{C}-2 \alpha$

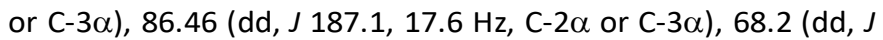

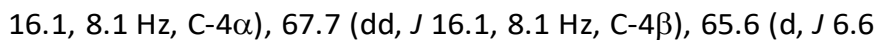

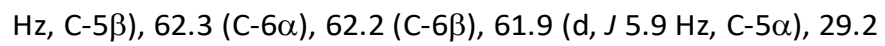
$\left(\mathrm{C}\left(\mathrm{CH}_{3}\right)_{2}-\alpha\right), 28.9\left(\mathrm{C}\left(\mathrm{CH}_{3}\right)_{2}-\beta\right), 18.3\left(\mathrm{C}\left(\mathrm{CH}_{3}\right)_{2}-\beta\right), 18.2\left(\mathrm{C}\left(\mathrm{CH}_{3}\right)_{2}-\alpha\right)$ ppm; ${ }^{19} \mathrm{~F} \mathrm{NMR}\left(376 \mathrm{MHz} \mathrm{CDCl}_{3}\right.$ ): (ratio $\alpha: \beta$ 7:1) $\delta-205.5--206.3$ (1F, m, F-2 $\beta$ or $F-3 \beta),-207.6--208.8(1 F, m, F-2 \beta$ or $F-3 \beta)$, $208.9--210.1$ (1F, m, F-2 $\alpha$ or F-3 $\alpha$ ), $-211.1--212.1$ (1F, m, F$2 \alpha$ or $\mathrm{F}-3 \alpha) \mathrm{ppm} ;{ }^{19} \mathrm{~F}\left\{{ }^{1} \mathrm{H}\right\}$ NMR $\left(471 \mathrm{MHz}, \mathrm{CDCl}_{3}\right):($ ratio $\alpha: \beta$ 7:1) $\delta-205.7(1 F, d, J 13.9 \mathrm{~Hz}, \mathrm{~F}-2 \beta$ or $\mathrm{F}-3 \beta),-208.0(1 \mathrm{~F}, \mathrm{~d}, J 14.0 \mathrm{~Hz}$, $\mathrm{F}-2 \beta$ or $\mathrm{F}-3 \beta),-209.4(1 \mathrm{~F}, \mathrm{~d}, J 13.6 \mathrm{~Hz}, \mathrm{~F}-2 \alpha$ or $\mathrm{F}-3 \alpha),-211.4(1 \mathrm{~F}$, d, J $13.6 \mathrm{~Hz}, \mathrm{~F}-2 \alpha$ or F-3 $\alpha$ ) ppm; MS (ESI+) (m/z) 247 [M+Na]+; HRMS (ESI+) for $\mathrm{C}_{9} \mathrm{H}_{14} \mathrm{~F}_{2} \mathrm{NaO}_{4}[\mathrm{M}+\mathrm{Na}]^{+}$calcd. 247.0757, found 247.0758.

Data for 2,3- $F_{2}$-galactofuranose acetal $17 \mathrm{c}$ : $[\alpha]_{\mathrm{D}}$ This compound could not be obtained in suitable purity. $\mathbf{R}_{\mathbf{f}} 0.45$ (hexane/acetone, 1:1); IR (neat) 3420 (m br), 2991 (m br), 2349 (m), 1221 (m), 1054 (s) cm ${ }^{-1} ;{ }^{1} \mathbf{H}$ NMR $\left(500 \mathrm{MHz}, \mathrm{CDCl}_{3}\right.$ ): (ratio minor/major 2:5) $\delta 5.58(1 \mathrm{H}, \mathrm{dd}, J 10.6,3.1 \mathrm{~Hz}, \mathrm{H}-1$ major), 5.39 (1H, dt, J 10.0, 4.3 Hz, H-1 minor), 5.25 (1H, ddt, J 54.6, 16.3, 4.4 $\mathrm{Hz}, \mathrm{H}-3$ minor), 5.09 (1H, dddd, 49.3, 14.0, 1.1, $0.5 \mathrm{~Hz}, \mathrm{H}-2$ major), 5.08 (1H, ddt, J 51.6, 20.6, $4.3 \mathrm{~Hz}, \mathrm{H}-2$ minor), $4.98(1 \mathrm{H}$, J 51.6, 20.3, 4.6, $1.1 \mathrm{~Hz}, \mathrm{H}-3$ major), 4.40 (1H, ddd, J 22.6, 5.7, $5.7 \mathrm{~Hz}, \mathrm{H}-4$ major), 4.33 (1H, dt, J 6.7, $5.6 \mathrm{~Hz}, \mathrm{H}-5$ major), 4.31 $(1 \mathrm{H}, J$ J 6.6, 3.7 Hz, H-5 minor), $4.14(1 \mathrm{H}$, ddd, J 24.0, 4.2, 3.8 Hz, $\mathrm{H}-4$ minor), $4.11(1 \mathrm{H}, \mathrm{dd}, J 8.7,6.7 \mathrm{~Hz}, \mathrm{H}-6$ major $), 4.09(1 \mathrm{H}, \mathrm{dd}$, J 8.4, $6.5 \mathrm{~Hz}, \mathrm{H}-6$ minor), 3.95 - $3.86\left(1 \mathrm{H}, \mathrm{m}, \mathrm{H}-6^{\prime}\right.$ minor), 3.89 (1H, dd, J 8.7, $5.8 \mathrm{~Hz}, \mathrm{H}-6^{\prime}$ major), $3.82(1 \mathrm{H}, \mathrm{d}, J 10.0 \mathrm{~Hz}, \mathrm{OH}$ major), $3.03(1 \mathrm{H}, \mathrm{d}, J 3.5 \mathrm{~Hz}, \mathrm{OH}$ minor), $1.49(3 \mathrm{H}, \mathrm{d}, J 0.6 \mathrm{~Hz}$ $\mathrm{C}\left(\mathrm{C}_{3}\right)_{2}$ minor), $1.47\left(3 \mathrm{H}, \mathrm{d}, J 0.6 \mathrm{~Hz} \mathrm{C}\left(\mathrm{C}_{3}\right)_{2}\right.$ major), 1.41 (3H, d, J $0.6 \mathrm{~Hz} \mathrm{C}\left(\mathrm{CH}_{3}\right)_{2}$ minor $), 1.39\left(3 \mathrm{H}, \mathrm{d}, J 0.6 \mathrm{~Hz} \mathrm{C}\left(\mathrm{CH}_{3}\right)_{2}\right.$ major $) \mathrm{ppm}$; ${ }^{13} \mathrm{C}$ NMR (100 MHz, $\mathrm{CDCl}_{3}$ ): (ratio minor/major 2:5) $\delta 110.4$ ( $\underline{\mathrm{C}}\left(\mathrm{CH}_{3}\right)_{2}$ minor), $110.3\left(\underline{\mathrm{C}}\left(\mathrm{CH}_{3}\right)_{2}\right.$ major), 100.0 (dd, J 35.9, $5.1 \mathrm{~Hz}$, C-1 major), 97.9 (dd, J 181.2, 27.1 Hz, C-2 major), 95.3 (dd, J 19.1 $\mathrm{Hz}, J 8.1 \mathrm{~Hz}, \mathrm{C}-1$ minor), 94.91 (dd, J 185.6, $25.7 \mathrm{~Hz}, \mathrm{C}-3$ minor), 94.89 (dd, J 185.6, 30.1 Hz, C-3 major), 93.6 (dd, J 195.1, 24.9 Hz, C-2 minor), 82.8 (dd, J 27.1, $2.9 \mathrm{~Hz}, \mathrm{C}-4$ major), 79.0 (dd, J 26.4, 7.3 Hz, C-4 minor), 75.2 (d, J 5.9 Hz, C-5 minor), 74.7 (d, J $5.1 \mathrm{~Hz}, \mathrm{C}-5$ major), 65.2 (C-6 minor and major), $26.3\left(\mathrm{C}\left(\mathrm{CH}_{3}\right)_{2}\right.$ major), $26.1\left(\mathrm{C}\left(\mathrm{CH}_{3}\right)_{2}\right.$ minor $), 25.3\left(\mathrm{C}\left(\mathrm{CH}_{3}\right)_{2}\right.$ minor $), 25.0\left(\mathrm{C}\left(\underline{C}_{3}\right)_{2}\right.$ major) ppm; ${ }^{19} \mathrm{~F}$ NMR (376 MHz, $\mathrm{CDCl}_{3}$ ): (ratio minor/major 2:5) $\delta$-193.9 (1F, dddd, J 51.4, 21.9, 14.3, 7.9 Hz, F-3 major), -195.6 (1F, ddt, J 49.0, 19.5, 8.1 Hz, F-2 major),-196.0 - -196.9 (1F, m, F3 minor), -208.4 (1F, ddd, J 52.0, 16.0, 7.2 Hz, F-2 minor) ppm; ${ }^{19} \mathrm{~F}\left\{{ }^{1} \mathrm{H}\right\}$ NMR (476 $\mathrm{MHz}, \mathrm{CDCl}_{3}$ ): (ratio minor/major 2:5) $\delta$-193.9 (1F, d, J 7.9 Hz, F-3 major), -195.6 (1F, d, J 8.1 Hz F-2 major),196.1 (1F, d, J $7.2 \mathrm{~Hz}, \mathrm{~F}-3$ minor), -208.4 (1F, d, J $7.2 \mathrm{~Hz}, \mathrm{~F}-2$ minor) ppm; MS (ESI+) (m/z) $247[\mathrm{M}+\mathrm{Na}]^{+}$; HRMS (ESI+) for $\mathrm{C}_{9} \mathrm{H}_{14} \mathrm{~F}_{2} \mathrm{NaO}_{4}[\mathrm{M}+\mathrm{Na}]^{+}$calcd. 247.0757, found 247.0758.

\section{5,6-Di-O-isopropylidene-2,3-dideoxy-2,3-difluoro-D- galactofuranose 17c (from 16d)}

Galactopyranose acetal $16 \mathrm{~d}(1.86 \mathrm{~g}, 8.30 \mathrm{mmol})$ was subjected to the same conditions as above to give, after column chromatography, the furanose acetal $\mathbf{1 7 c}$ as a pale yellow oil (905, $4.04 \mathrm{mmol}, 49 \%$, combined yield of $60 \%$ over 2 steps), alongside with the recovered starting material $16 \mathrm{c}(837 \mathrm{mg}$, $3.73 \mathrm{mmol}, 45 \%$, combined yield of $20 \%$ over 2 steps).

\section{1-O-Acetyl-5,6-di-O-isopropylidene-2,3-dideoxy-2,3-difluoro-D- galactofuranose $17 \mathrm{~d}$}

To a solution of 17c $(469 \mathrm{mg}, 2.09 \mathrm{mmol})$ in $\mathrm{CH}_{2} \mathrm{Cl}_{2}(10 \mathrm{~mL})$ at 0 ${ }^{\circ} \mathrm{C}$ was successively added DMAP ( $38 \mathrm{mg}, 0.31 \mathrm{mmol}, 15 \mathrm{~mol} \%$ ) and $\mathrm{Ac}_{2} \mathrm{O}(275 \mu \mathrm{L}, 2.93 \mathrm{mmol})$. The mixture was stirred at $\mathrm{rt}$ for $3 \mathrm{~h}$, before quenching with a sat. aq. solution of $\mathrm{NaHCO}_{3}(10$ $\mathrm{mL}$ ). Phases were separated and the aqueous layer was extracted with $\mathrm{CH}_{2} \mathrm{Cl}_{2}(10 \mathrm{~mL})$, dried over $\mathrm{MgSO}_{4}$, and concentrated. Filtration over a pad of silica gel (petroleum ether/acetone 8:2) gave the protected intermediate $17 \mathrm{~d}$ as a colourless oil $(516 \mathrm{mg}, 1.93 \mathrm{mmol}, 92 \%$, ratio minor/major 1:10), which was directly submitted to the next reaction. $\mathbf{R}_{\boldsymbol{f}} 0.40$ (hexane/acetone, 7:3); ${ }^{1} \mathbf{H}$ NMR (selected peaks, major anomer only) $\left(400 \mathrm{MHz}, \mathrm{CDCl}_{3}\right): \delta 6.36(1 \mathrm{H}, \mathrm{d}, J 10.5 \mathrm{~Hz}, \mathrm{H}-1), 5.14(1 \mathrm{H}$, br. dd, J 48.7, $13.0 \mathrm{~Hz}, \mathrm{H}-2$ ), 5.11 (br. ddd, J 51.8, 19.6, 3.42 Hz, $\mathrm{H}-3), 4.40(1 \mathrm{H}, \mathrm{br} . \mathrm{dt}, J 15.9,4.7 \mathrm{~Hz}, \mathrm{H}-4), 4.38-4.33(1 \mathrm{H}, \mathrm{m}, \mathrm{H}-$ 5), $4.09(1 \mathrm{H}, \mathrm{dd}, J$ J $.0,6.9 \mathrm{~Hz}, \mathrm{H}-6), 3.89(1 \mathrm{H}, \mathrm{dd}, J$ 9.0, $5.4 \mathrm{~Hz}, \mathrm{H}-$ $\left.6^{\prime}\right), 2.13\left(3 \mathrm{H}, \mathrm{s}, \mathrm{COC} \underline{H}_{3}\right), 1.46\left(3 \mathrm{H}, \mathrm{s}, \mathrm{C}\left(\mathrm{CH}_{3}\right)_{2}\right), 1.38\left(3 \mathrm{H}, \mathrm{s}, \mathrm{C}\left(\mathrm{CH}_{3}\right)_{2}\right)$ ppm; ${ }^{13} \mathrm{C}$ NMR $\left(100 \mathrm{MHz}, \mathrm{CDCl}_{3}\right): \delta 169.1\left(\mathrm{COCH}_{3}\right), 110.2$ $\left(\underline{C}\left(\mathrm{CH}_{3}\right)_{2}\right), 98.6(\mathrm{dd}, J 37.4,4.4 \mathrm{~Hz}, \mathrm{C}-1), 97.0(\mathrm{dd}, J 183.4,29.3 \mathrm{~Hz}$, C-2 or C-3), 94.1 (dd, J 185.6, $30.8 \mathrm{~Hz}, \mathrm{C}-3$ or C-2), 84.3 (dd, J 27.1, 2.2 Hz, C-4), 74.0 (d, J $5.1 \mathrm{~Hz}, \mathrm{C}-5), 65.1$ (d, J $2.2 \mathrm{~Hz}, \mathrm{C}-6)$, $26.2\left(\left(\mathrm{C}\left(\mathrm{CH}_{3}\right)_{2}\right), 25.0\left(\left(\mathrm{C}\left(\mathrm{C}_{3}\right)_{2}\right)\right.\right.$ ppm ${ }^{19} \mathrm{~F}$ NMR $\left(376 \mathrm{MHz}, \mathrm{CDCl}_{3}\right)$ : $\delta$-192.8 (1F, dddd, J 51.6, 24.7, 13.9, 8.7 Hz, F-3 major), -195.0 (1F, ddt, J 48.5, 19.1, 8.7 Hz, F-2 major), -202.5 (1F, dtd, J 55.9, 19.3, $6.9 \mathrm{~Hz}, \mathrm{~F}-2$ minor or F-3 minor), -208.4 (1F, m, F-2 minor or F-3 minor) ppm ${ }^{19} \mathrm{~F}\left\{{ }^{1} \mathrm{H}\right\}$ NMR (376 MHz, $\left.\mathrm{CDCl}_{3}\right): \delta-192.8(1 \mathrm{~F}, \mathrm{~d}, J$ 8.7 Hz, F-3 major), -194.8 (1F, d, J 8.7 Hz, F-2 major), -202.5 (1F, d, J $7.6 \mathrm{~Hz}, \mathrm{~F}-2$ minor or F-3 minor), $-208.4(1 \mathrm{~F}, \mathrm{~d}, J 7.6 \mathrm{~Hz}, \mathrm{~F}-2$ minor or $\mathrm{F}-3$ minor) ppm; HRMS (ESI+) for $\mathrm{C}_{11} \mathrm{H}_{16} \mathrm{~F}_{2} \mathrm{NaO}_{5}$ $[\mathrm{M}+\mathrm{Na}]^{+}$calcd 289.0863, found 289.0854 .

\section{1,5,6-Tri-O-acetyl-2,3-dideoxy-2,3-difluoro-D-galactofuranose 17b}

Compound 17d (480 mg, $1.80 \mathrm{mmol}$ ) was dissolved in $\mathrm{CH}_{2} \mathrm{Cl}_{2}$ (8 $\mathrm{mL}$ ), after which $\mathrm{Ac}_{2} \mathrm{O}(641 \mu \mathrm{L}, 6.83 \mathrm{mmol}), \mathrm{Bi}(\mathrm{OTf})_{3}(45 \mathrm{mg}$, $0.068 \mathrm{mmol}, 3.8 \mathrm{~mol} \%)$ and $\mathrm{H}_{2} \mathrm{O}(40 \mu \mathrm{L}, 2.29 \mathrm{mmol})$ were added in this order. The reaction was stirred at $\mathrm{rt}$ for $2 \mathrm{~h}$. The reaction was quenched with a sat. aq. solution of $\mathrm{NaHCO}_{3}(10 \mathrm{~mL})$. The phases were separated and the organic layer was washed with a sat. aq. solution of $\mathrm{NaHCO}_{3}(1 \times 10 \mathrm{~mL})$. The combined aqueous layers were then extracted with DCM $(110 \mathrm{~mL})$. The combined organic layers were combined, dried over $\mathrm{MgSO}_{4}$, and concentrated in vacuo and then purified by column chromatography (silica, petroleum ether/acetone, 8:2) afforded 
17b as a yellow oil (500 mg, $1.61 \mathrm{mmol}, 89 \%$, minor/major 1:9). IR (neat) 2951 (w), 1743 (s), 1370 (m), 1216 (s), 1017 (m, br.) $\mathrm{cm}^{-1} ;{ }^{1} \mathbf{H}$ NMR $\left(400 \mathrm{MHz}, \mathrm{CDCl}_{3}\right): \delta 6.36(1 \mathrm{H}, \mathrm{d}, J 10.8 \mathrm{~Hz}, \mathrm{H}-1$ major), $6.29(1 \mathrm{H}, \mathrm{d}, J 3.9 \mathrm{~Hz}, \mathrm{H}-1$ minor), $5.38(1 \mathrm{H}, \mathrm{dt}, J 6.7,4.2$ $\mathrm{Hz}, \mathrm{H}-5$ major), $5.35-5.16(3 \mathrm{H}, \mathrm{m}, \mathrm{H}-2$ minor, $\mathrm{H}-3$ minor, $\mathrm{H}-5$ minor), 5.10 (1H, dd, J 48.4, $12.5 \mathrm{~Hz}, \mathrm{H}-3$ major), 5.07 (1H, dddt, J 50.9, 17.6, 3.7, $1.1 \mathrm{~Hz}, \mathrm{H}-2$ major), $4.53(1 \mathrm{H}, \mathrm{dt}$, J 24.7, $3.7 \mathrm{~Hz}$, $\mathrm{H}-4$ major), $4.36-4.18$ (2H, m, H-4 minor, $\mathrm{H}-6$ minor $), 4.31(1 \mathrm{H}$, dd, J 12.0, 4.4 Hz, H-6 major), $4.21(1 \mathrm{H}$, dd, J 11.7, 6.7 Hz, H-6' major), $4.13\left(1 \mathrm{H}, \mathrm{dd}, J 12.2,6.1 \mathrm{~Hz}, \mathrm{H}-6^{\prime}\right.$ minor), $2.14(3 \mathrm{H}, \mathrm{s}$, $\mathrm{COC}_{\underline{3}}$ minor), $2.11\left(3 \mathrm{H}, \mathrm{s}, \mathrm{COC}_{3} \underline{3}\right.$ major), $2.10\left(3 \mathrm{H}, \mathrm{s}, \mathrm{COC}_{3}\right.$

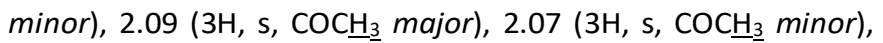
$2.05\left(3 \mathrm{H}, \mathrm{s}, \mathrm{COC}_{3}\right.$ major) ppm; ${ }^{13} \mathrm{C}$ NMR $\left(101 \mathrm{MHz}, \mathrm{CDCl}_{3}\right): \delta$ ppm 170.41 ( $\mathrm{COCH}_{3}$ major), 170.38 ( $\mathrm{COCH}_{3}$ minor), 170.3 ( $\mathrm{COCH}_{3}$ major), 169.9 ( $\mathrm{COCH}_{3}$ minor), 169.1 ( $\underline{\mathrm{COCH}}_{3}$ minor), 169.0 ( $\mathrm{COCH}_{3}$ major), 98.6 (dd, J 37.4, 3.7 Hz, C-1 major), 96.5 (dd, J 183.4, 29.3 Hz, C-3 major), 94.1 (dd, J 185.6, 31.5 Hz, C-2 major), 93.6 (dd, J 189.3, 24.2 Hz, C-2 minor or C-3 minor), 92.4 (dd, J 17.6, 9.5 Hz, C-1 minor), 92.0 (dd, J 203.2, $24.2 \mathrm{~Hz}, \mathrm{C}-2$ minor or C-3 minor), 83.2 (dd, J 28.6, $1.5 \mathrm{~Hz}, \mathrm{C}-4$ major), 78.9 (dd, J 27.5, 8.4 Hz, C-4 minor), 70.3 (d, J 5.1 Hz, C-5 minor), 68.9 (d, J $6.6 \mathrm{~Hz}, \mathrm{C}-5$ major), 62.2 (C-6 major), 61.9 (C-6 minor), 20.93 $\left(\mathrm{COCH}_{3}\right.$ minor), 20.87 ( $\mathrm{COCH}_{3}$ major), $20.72\left(\mathrm{COCH}_{3}\right.$ minor), $20.67\left(\mathrm{COCH}_{3}\right.$ major), $20.66\left(\mathrm{COCH}_{3}\right.$ major $), 20.61\left(\mathrm{COCH}_{3}\right.$ minor) ppm; ${ }^{19} \mathrm{~F}$ NMR $\left(376 \mathrm{MHz}, \mathrm{CDCl}_{3}\right): \delta-192.0(1 \mathrm{~F}$, dddd, $J$ 51.2, 25.1, 13.0, 9.5 Hz, F-2 major), -196.1 (1F, ddt, J 48.6, 17.3, $9.5 \mathrm{~Hz}, \mathrm{~F}-3$ major), -200.1 - -200.8 (1F, m, F-2 minor or F-3 minor), $-207.0--209.4(1 \mathrm{~F}, \mathrm{~m}, \mathrm{~F}-2$ minor or $\mathrm{F}-3$ minor) ppm ${ }^{19} \mathrm{~F}\left\{{ }^{1} \mathrm{H}\right\}$ NMR $\left(376 \mathrm{MHz}, \mathrm{CDCl}_{3}\right): \delta-191.9(1 \mathrm{~F}, \mathrm{~d}, J 6.94 \mathrm{~Hz}, \mathrm{~F}-2$ major), -196.0 (1F, d, J $6.9 \mathrm{~Hz}, \mathrm{~F}-3$ major), $-200.4(1 \mathrm{~F}, \mathrm{~d}, J 6.9 \mathrm{~Hz}$, F-2 minor or F-3 minor), $-208.1(1 \mathrm{~F}, \mathrm{~d}, J 6.9 \mathrm{~Hz}, \mathrm{~F}-2$ minor or F-3 minor) ppm; MS (ESI+) (m/z) $333[\mathrm{M}+\mathrm{Na}]^{+}$; HRMS (ESI+) for $\mathrm{C}_{12} \mathrm{H}_{16} \mathrm{~F}_{2} \mathrm{NaO}_{7}[\mathrm{M}+\mathrm{Na}]^{+}$calcd 333.0761 , found 333.0755 .

\section{Conflicts of interest}

There are no conflicts to declare.

\section{Acknowledgements}

This project has been funded by the Industrial Biotechnology Catalyst (Innovate UK, BBSRC, EPSRC, BB/M028941/1) to support the translation, development and commercialization of innovative Industrial Biotechnology processes. We also thank the EPSRC (core capability EP/K039466/1) for funding.

\section{Notes and references}

1 I. P. Street, C. R. Armstrong and S. G. Withers, Biochemistry, 1986, 25, 6021-6027.

2 C. P. J. Glaudemans, Chem. Rev., 1991, 91, 25-33.

3 A. Arda and J. Jimenez-Barbero, Chem Commun (Camb), 2018, 54, 4761-4769.

4 S. Wagner, C. Mersch and A. Hoffmann-Roeder, Chem. Eur. J., 2010, 16, 7319-7330.

5 G. R. Morais, R. A. Falconer and I. Santos, Eur. J. Org. Chem., 2013, DOI: 10.1002/ejoc.201201457, 1401-1414.
6 S. M. Ametamey, M. Honer and P. A. Schubiger, Chem. Rev., 2008, 108, 1501-1516.

7 J.-H. Kim, R. Resende, T. Wennekes, H.-M. Chen, N. Bance, S. Buchini, A. G. Watts, P. Pilling, V. A. Streltsov, M. Petric, R. Liggins, S. Barrett, J. L. McKimm-Breschkin, M. Niikura and S. G. Withers, Science, 2013, 340, 71-75.

8 S. G. Withers, K. Rupitz and I. P. Street, J. Biol. Chem., 1988, 263, 7929-7932.

9 C. Tysoe and S. G. Withers, Curr. Top. Med. Chem., 2014, 14, 865-874.

10 J. P. Ribeiro, T. Diercks, J. Jimenez-Barbero, S. Andre, H. J. Gabius and F. J. Canada, Biomolecules, 2015, 5, 3177-3192.

11 T. Diercks, A. S. Infantino, L. Unione, J. Jimenez-Barbero, S. Oscarson and H. J. Gabius, Chem. Eur. J., 2018, 24, 1576115765.

12 H. W. Kim, P. Rossi, R. K. Shoemaker and S. G. DiMagno, J. Am. Chem. Soc., 1998, 120, 9082-9083.

13 J. C. Biffinger, H. W. Kim and S. G. DiMagno, Chembiochem, 2004, 5, 622-627.

14 S. Bresciani, T. Lebl, A. M. Z. Slawin and D. O'Hagan, Chem. Commun., 2010, 46, 5434-5436.

15 V. Denavit, D. Lainé, J. St-Gelais, P. A. Johnson and D. Giguère, Nat. Commun., 2018, 9, 4721.

16 L. Quiquempoix, Z. Wang, J. Graton, P. G. Latchem, M. Light, J.-Y. Le Questel, and B. Linclau, J. Org. Chem. 2019, 84, 58995906.

17 V. Denavit, D. Laine, C. Bouzriba, E. Shanina, E. Gillon, S. Fortin, C. Rademacher, A. Imberty and D. Giguere, Chemistry, 2019, 25, 4478-4490.

18 R. S. Timofte and B. Linclau, Org. Lett., 2008, 10, 3673-3676.

19 S. Golten, C. Q. Fontenelle, R. S. Timofte, L. Bailac, M. Light, M. Sebban, H. Oulyadi and B. Linclau, J. Org. Chem., 2016, 81, 4434-4453.

20 I. N'Go, S. Golten, A. Ardá, J. Cañada, J. Jiménez-Barbero, B. Linclau and S. P. Vincent, Chem. Eur. J., 2014, 20, 106-112.

21 K. E. van Straaten, J. R. Kuttiyatveetil, C. M. Sevrain, S. A. Villaume, J. Jimenez-Barbero, B. Linclau, S. P. Vincent and D. A. Sanders, J. Am. Chem. Soc., 2015, 137, 1230-1244.

22 C. Q. Fontenelle, D. Shishmarev, P. W. Kuchel and B. Linclau, Trends Carbohydr. Res., 2017, 9, 28-33.

23 Š. Horník, L. Červenková Št́astná, P. Cuřínová, J. Sýkora, K. Káňová, R. Hrstka, I. Císařová, M. Dračínský and J. Karban, Beilst. J. Org. Chem., 2016, 12, 750-759.

24 L. Mtashobya, L. Quiquempoix and B. Linclau, J. Fluorine Chem., 2015, 171, 92-96.

25 B. Linclau, Z. Wang, G. Compain, V. Paumelle, C. Q. Fontenelle, N. Wells and A. Weymouth-Wilson, Angew. Chem. Int. Ed. Engl., 2016, 55, 674-678.

26 J. Adamson and D. M. Marcus, Carbohydr. Res., 1972, 22, 257264.

27 W. Korytnyk, S. Valentekovic-Horvath and C. R. Petrie, Tetrahedron, 1982, 38, 2547-2550.

28 M. Diksic and D. Jolly, Carbohydr. Res., 1986, 153, 17-24.

29 C. C. Geilen, N. Loch, W. Reutter, K. Seppelt and F. Oberdorfer, Tetrahedron Lett., 1992, 33, 2435-2438.

30 M. Albert, K. Dax and J. Ortner, Tetrahedron, 1998, 54, 48394848.

31 L. Barbieri, V. Costantino, E. Fattorusso, A. Mangoni, N. Basilico, M. Mondani and D. Taramelli, Eur. J. Org. Chem., 2005, 2005, 3279-3285.

32 J. Karban, I. Cisarova, T. Strasak, L. C. Stastna and J. Sykora, Org. Biomol. Chem., 2012, 10, 394-403.

33 M. Cerny, I. Buben and J. Pacak, Collect. Czech. Chem. Commun., 1963, 28, 1569-1578.

34 A. Furst and P. A. Plattner, Helv. Chim. Acta, 1949, 32, 275283.

35 J. Halbych, T. Trnka and M. Cerny, Collect. Czech. Chem. Commun., 1973, 38, 2151-2166. 
36 T. Katagiri and K. Uneyama, J. Fluorine Chem., 2000, 105, 285293.

37 F. G. Bordwell and W. T. Brannen, J. Am. Chem. Soc., 1964, 86, 4645-4650.

38 C. Wong, R. J. Griffin, I. R. Hardcastle, J. S. Northen, L.-Z. Wang and B. T. Golding, Org. Biomol. Chem., 2010, 8, 2457-2464.

39 J. Hine and W. H. Brader, J. Am. Chem. Soc., 1953, 75, 39643966.

40 V. Wray, J. Chem. Soc., Perkin Trans. 2, 1976, DOI: 10.1039/P29760001598, 1598-1605.

41 M. Zottola, B. V. Rao and B. Fraser-Reid, J. Chem. Soc., Chem. Commun., 1991, DOI: 10.1039/C39910000969, 969-970.

42 C. Altona and C. A. G. Haasnoot, Organic Magnetic Resonance, 1980, 13, 417-429.

43 H. Amarasekara, S. Dharuman, T. Kato and D. Crich, J. Org. Chem., 2018, DOI: 10.1021/acs.joc.7b02891.

44 P. Sarda, F. C. Escribano, R. José Alves, A. Olesker and G. Lukacs, J. Carbohydr. Chem., 1989, 8, 115-123.

45 P. I. Abronina, N. N. Malysheva, V. V. Litvinenko, A. I. Zinin, N. G. Kolotyrkina and L. O. Kononov, Org. Lett., 2018, DOI: 10.1021/acs.orglett.8b02424.

46 S. Manhas and M. S. Taylor, J. Org. Chem., 2017, 82, 1140611417.

47 N. Nifantiev, V. Krylov, D. Argunov, D. Vinnitskiy, A. Gerbst, N. Ustyuzhanina and A. Dmitrenok, Synlett, 2016, 27, 1659-1664.

48 D. A. Argunov, V. B. Krylov and N. E. Nifantiev, Org. Lett., 2016, 18, 5504-5507.

49 V. B. Krylov, D. A. Argunov, D. Z. Vinnitskiy, S. A. Verkhnyatskaya, A. G. Gerbst, N. E. Ustyuzhanina, A. S. Dmitrenok, J. Huebner, O. Holst, H. C. Siebert and N. E. Nifantiev, Chemistry, 2014, 20, 16516-16522.

50 M. Giordano and A. Iadonisi, J. Org. Chem., 2014, 79, 213-222.

51 J.-S. Zhu, N. E. McCormick, S. C. Timmons and D. L. Jakeman, The Journal of Organic Chemistry, 2016, 81, 8816-8825.

52 Z. Hricovíniová, Synthesis, 2001, 2001, 0751-0754.

53 B. Linclau, A. J. Boydell, P. J. Clarke, R. Horan and C. Jacquet, J. Org. Chem., 2003, 68, 1821-1826.

54 Q.-P. Wu, M.-X. Zhou, X.-D. Xi, D. Song, Y. Wang, H.-X. Liu, Y.Z. Li and Q.-S. Zhang, Tetrahedron Lett., 2008, 49, 2714-2718. 